\title{
Integrable Theory of the Perturbation Equation
}

\author{
Wen-Xiu Ma* and Benno Fuchssteiner \\ FB Mathematik-Informatik, Universität Paderborn, \\ D-33098 Paderborn, Germany
}

\begin{abstract}
An integrable theory is developed for the perturbation equations engendered from small disturbances of solutions. It includes various integrable properties of the perturbation equations: hereditary recursion operators, master symmetries, linear representations (Lax and zero curvature representations) and Hamiltonian structures etc. and provides us a method to generate hereditary operators, Hamiltonian operators and symplectic operators starting from the known ones. The resulting perturbation equations give rise to a sort of integrable coupling of soliton equations. Two examples (MKdV hierarchy and KP equation) are carefully carried out.
\end{abstract}

\section{Introduction}

Integrable nonlinear wave or evolution equations (for instance, KdV, NLS, SG and KP equations) are ideal mathematical models of real physical phenomena although they play an outstanding role in physical problems. Therefore for these equations we often need to take into account the effect of small perturbation so that their applicability may be extended to higher order nonlinearity or larger amplitude waves. There are mainly two kinds of perturbation worthy studying for integrable equations. The one is the perturbation situation of integrable equations themselves and the other one, the perturbation situation of solutions of the original unperturbed integrable equations. They all can provide approximate solutions to real physical problems.

In the context of soliton perturbation, what one considers usually is the first kind of perturbation situation. There have been quiet a few of powerful techniques for dealing with this kind of the perturbation (see [1] [2] [3] [4] [5] and references therein). Various perturbed cases of integrable equations have been considered, including the perturbed $\mathrm{KdV}$

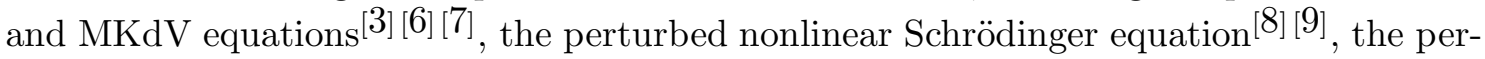
turbed Burgers equation [10] and the perturbed Benjamin-Ono equation [11] etc. A detailed

\footnotetext{
* On leave of absence from Institute of Mathematics, Fudan University, Shanghai 200433, P. R. of China; Current Fax: 0049-5251-603836 and Email: wenxiuma@uni-paderborn.de
} 
survey for this kind of perturbation theory based upon the inverse scattering transformation was provided by Kivshar and Malomed [12]. However there don't exist so many works devoted to the second kind of perturbation situation. Among them there are the following several works. Tamizhmani and Lakshmanan considered the complete integrability of the perturbed equations by small disturbance of solutions of KdV equation 13 . We analyzed the similar perturbation situation of the whole $\mathrm{KdV}$ integrable hierarchy and pointed out a mistake in Ref. 13. for the Hamiltonian structure 14. Recently it has been observed by Kraenkel et al. that a multiple time scale expansion may relate the solutions of long surface water-waves and the Boussinesq equation to KdV integrable hierarchy 15.16.

In this paper we would like to consider the second kind of perturbation. Mathematically, this kind of perturbation yields interesting results. For example, we shall show that it preserves complete integrability. In other words, the equations generated by the perturbation are still integrable and thus give rise to new examples of integrable equations. Moreover they are all special integrable coupling of the original integrable equations.

We now introduce our notation and conception. Some notation comes from Refs. [17], [18], 19]. Let $M=M(u)$ be a suitable manifold possessing a manifold variable $u$ (we write $u$ as a column vector) and $\hat{M}_{N}=\hat{M}_{N}\left(\hat{\eta}_{N}\right)$ be another suitable manifold possessing a manifold variable $\hat{\eta}_{N}=\left(\eta_{0}^{T}, \eta_{1}^{T}, \cdots, \eta_{N}^{T}\right)^{T}, N \geq 1$, where $\eta_{i}, 0 \leq i \leq N$, are column vectors and $T$ means the transpose of matrices. Assume that $T(M), T\left(\hat{M}_{N}\right)$ denote the tangent bundles on $M$ and $\hat{M}_{N}, T^{*}(M), T^{*}\left(\hat{M}_{N}\right)$ denote the cotangent bundles on $M$ and $\hat{M}_{N}$, and $C^{\infty}(M), C^{\infty}\left(\hat{M}_{N}\right)$ denote the spaces of smooth functions on $M$ and $\hat{M}_{N}$, respectively. Throughout this paper we require that the column vector $\eta_{i}(0 \leq i \leq N)$ has the same dimension as the column vector $u$. Therefore we have $\eta_{i} \in \mathbb{R}^{q}(0 \leq i \leq N)$ when $u \in \mathbb{R}^{q}$. Further let $T_{s}^{r}(M)$ be the s-times co- and r-times contravariant tensor bundle and $\left(T_{s}^{r}\right)_{u}(M)$, the space of s-times co- and r-times contravariant tensors at $u \in M$. We use $X(u)$ (not $\left.X\right|_{u}$ ) to denote a tensor of $X \in T_{s}^{r}(M)$ at $u \in M$ but sometimes we omit the point $u$ for convenience while there is no confusion of the symbols. Note that linear maps $\Phi: T(M) \rightarrow T(M), \Psi: T^{*}(M) \rightarrow T^{*}(M), J: T^{*}(M) \rightarrow T(M), \Theta: T(M) \rightarrow T^{*}(M)$ may be identified with the second degree tensor fields $T_{\Phi} \in T_{1}^{1}(M), T_{\Psi} \in T_{1}^{1}(M), T_{J} \in$ $T_{0}^{2}(M), T_{\Theta} \in T_{2}^{0}(M)$ by the following relations 19

$$
\begin{aligned}
& T_{\Phi}(u)(\alpha(u), K(u))=<\alpha(u), \Phi(u) K(u)>, \alpha \in T^{*}(M), K \in T(M), \\
& T_{\Psi}(u)(\alpha(u), K(u))=<\Psi(u) \alpha(u), K(u)>, \alpha \in T^{*}(M), K \in T(M), \\
& T_{J}(u)(\alpha(u), \beta(u))=<\alpha(u), J(u) \beta(u)>, \alpha, \beta \in T^{*}(M), \\
& T_{\Theta}(u)(K(u), S(u))=<\Theta(u) K(u), S(u)>, K, S \in T(M),
\end{aligned}
$$

where $\langle\cdot, \cdot\rangle$ denotes the duality between cotangent vectors and tangent vectors.

A basic tool to handle various kinds of tensor fields is the conception of the Gateaux derivative. For a tensor fields $X \in T_{s}^{r}(M)$, its Gateaux derivative at the direction $S \in$ 
$T(M)$ is defined by

$$
X^{\prime}(u)[S(u)]=\left.\frac{\partial X(u+\varepsilon S(u))}{\partial \varepsilon}\right|_{\varepsilon=0} .
$$

For four kinds of operators between the tangent bundle and the cotangent bundle, their Gateaux derivatives may be given similarly or by means of their tensor fields. The commutator of two vector fields $K, S \in T(M)$ and the adjoint map $\operatorname{ad}_{K}$ are defined by

$$
[K, S](u)=K^{\prime}(u)[S(u)]-S^{\prime}(u)[K(u)], \operatorname{ad}_{K} S=[K, S] .
$$

The conjugate operator of an operator between the tangent bundle and the cotangent bundle is established in terms of the duality between cotangent vectors and tangent vectors. For example, we may calculate the conjugate operator $\Phi^{\dagger}: T^{*}(M) \rightarrow T^{*}(M)$ of an operator $\Phi: T(M) \rightarrow T(M)$ through

$$
<\Phi^{\dagger}(u) \alpha(u), K(u)>=<\alpha(u), \Phi(u) K(u)>, \alpha \in T^{*}(M), K \in T(M) .
$$

If an operator $J: T^{*}(M) \rightarrow T(M)$ (or $\Theta: T(M) \rightarrow T^{*}(M)$ ) plus its conjugate operator equals to zero, then it is called skew-symmetric.

Definition 1.1 For $H \in C^{\infty}(M)$, its variational derivative $\frac{\delta H}{\delta u} \in T^{*}(M)$ is defined by

$$
<\frac{\delta H}{\delta u}(u), K(u)>=<\frac{\delta H(u)}{\delta u}, K(u)>=H^{\prime}(u)[K(u)], K \in T(M) .
$$

If for $\gamma \in T^{*}(M)$ there exists $H \in C^{\infty}(M)$ so that

$$
H^{\prime}(u)[K(u)]=<\gamma(u), K(u)>\text {, for all } K \in T(M),
$$

holds, then $\gamma \in T^{*}(M)$ is called a gradient field with a potential $H \in C^{\infty}(M)$.

A cotangent vector field $\gamma \in T^{*}(M)$ is a gradient field iff

$$
\begin{aligned}
& (d \gamma)(u)(K(u), S(u)) \\
& :=<\gamma^{\prime}(u)[K(u)], S(u)>-<\gamma^{\prime}(u)[S(u)], K(u)>=0, K, S \in T(M) .
\end{aligned}
$$

If $\gamma \in T^{*}(M)$ is gradient, then its potential $H \in C^{\infty}(M)$ is given by

$$
H(u)=\int_{0}^{1}<\gamma(\lambda u), u>d \lambda .
$$

Definition 1.2 A linear operator $\Phi: T(M) \rightarrow T(M)$ is called a recursion operator of $u_{t}=K(u), K \in T(M)$, if we have for all $S \in T(M)$ and $u \in M$

$$
\frac{\partial \Phi(u)}{\partial t} S(u)+\Phi^{\prime}(u)[K(u)] S(u)-K^{\prime}(u)[\Phi(u) S(u)]+\Phi(u) K^{\prime}(u)[S(u)]=0 .
$$


Evidently a recursion operator $\Phi: T(M) \rightarrow T(M)$ of $u_{t}=K(u)$ maps symmetries into new symmetries of $u_{t}=K(u)$.

Definition 1.3 A linear operator $\Phi: T(M) \rightarrow T(M)$ is called a hereditary operator 21. if the following equality holds

$$
\begin{aligned}
& \Phi^{\prime}(u)[\Phi(u) K(u)] S(u)-\Phi(u) \Phi^{\prime}(u)[K(u)] S(u) \\
& \quad-\Phi^{\prime}(u)[\Phi(u) S(u)] K(u)+\Phi(u) \Phi^{\prime}(u)[S(u)] K(u)=0
\end{aligned}
$$

for all vector fields $K, S \in T(M)$.

When an evolution equation $u_{t}=K(u)$ possesses a time-independent hereditary recursion operator $\Phi$, a hierarchy of vector fields $\Phi^{n} K, n \geq 0$, are all symmetries and commute with each other. If the conjugate operator $\Psi=\Phi^{\dagger}$ of the hereditary operator $\Phi: T(M) \rightarrow T(M)$ maps a gradient field $\gamma \in T^{*}(M)$ into another gradient field, then $\Psi^{n} \gamma, n \geq 0$, are all gradient fields 22 23.

Definition 1.4 A linear skew-symmetric operator $J: T^{*}(M) \rightarrow T(M)$ is called a Hamiltonian operator if for all $\alpha, \beta, \gamma \in T^{*}(M)$ we have

$$
<K(u), J^{\prime}(u)[J(u) S(u)] T(u)>+\operatorname{cycle}(K, S, T)=0 .
$$

The corresponding Poisson bracket is defined by

$$
\left\{H_{1}, H_{2}\right\}_{J}(u)=<\frac{\delta H_{1}}{\delta u}(u), J(u) \frac{\delta H_{2}}{\delta u}(u)>, H_{1}, H_{2} \in C^{\infty}(M) .
$$

A pair of operators $J, M: T^{*}(M) \rightarrow T(M)$ is called a Hamiltonian pair if $J+c M$ is always Hamiltonian for any constant $c$.

When $J: T^{*}(M) \rightarrow T(M)$ is Hamiltonian, we have 22, 23

$$
J \frac{\delta}{\delta u}\left\{H_{1}, H_{2}\right\}_{J}=\left[J \frac{\delta H_{1}}{\delta u}, J \frac{\delta H_{2}}{\delta u}\right], H_{1}, H_{2} \in C^{\infty}(M) .
$$

Moreover if $J, M: T^{*}(M) \rightarrow T(M)$ is a Hamiltonian pair and $J$ is invertible, then $\Phi=$ $M J^{-1}: T(M) \rightarrow T(M)$ is hereditary 17 .

Definition 1.5 A linear skew-symmetric operator $\Theta: T(M) \rightarrow T^{*}(M)$ is called a symplectic operator if for all $K, S, T \in T(M)$ we have

$$
<K(u), \Theta^{\prime}(u)[S(u)] T(u)>+\operatorname{cycle}(K, S, T)=0 .
$$

If $\Theta: T(M) \rightarrow T^{*}(M)$ is a symplectic operator, then its second degree tensor field $T_{\Theta} \in$ $T_{2}^{0}(M)$ may be expressed as

$$
T_{\Theta}=d \gamma \text { with }<\gamma(u), K(u)>=\int_{0}^{1}<\Theta(\lambda u) \lambda u, K(u)>d \lambda, K \in T(M),
$$

where $d \gamma$ is defined by (1.3). It is not difficult to prove that the inverse of symplectic operators are Hamiltonian if they exist and vice verse. 
Definition 1.6 An evolution equation $u_{t}=K(u), K \in T(M)$, is called a Hamiltonian equation if there exists a function $H \in C^{\infty}(M)$ so that

$$
u_{t}=K(u)=J(u) \frac{\delta H}{\delta u}(u) .
$$

It is called a bi-Hamiltonian equation if there exist two functions $H_{1}, H_{2} \in C^{\infty}(M)$ and a Hamiltonian pair $J, M: T^{*}(M) \rightarrow T(M)$ so that

$$
u_{t}=K(u)=J(u) \frac{\delta H_{1}}{\delta u}(u)=M \frac{\delta H_{2}}{\delta u}(u) .
$$

There is another kind of Hamiltonian equations, which may be defined by symplectic operators. However, the above definition is more advantageous. For a bi-Hamiltonian

equation above, there exist several beautiful characteristics in the aspects of algebra and geometry22 23.

In this paper, we shall analyze the perturbation equations of the evolution equation

$$
u_{t}=K(u), K \in T(M)
$$

under the perturbation series

$$
\hat{u}_{N}=\sum_{i=0}^{N} \varepsilon^{i} \eta_{i}, \hat{\eta}_{N}=\left(\eta_{0}^{T}, \eta_{1}^{T} \cdots, \eta_{N}^{T}\right)^{T}, N \geq 1
$$

and their integrable properties. The paper is organized as follows. In Section 2 we propose three useful theorems to generate Hereditary operators, Hamiltonian operators and symplectic operators from the perturbation series (1.12) in a natural and explicit way. We show in Section 3 that the perturbation (1.12) preserves complete integrability, i.e. we want to show that the perturbation equations are still integrable equations provided that the equation under consideration is integrable. In Section 4, we apply the resulting theory to MKdV hierarchy and KP equation as illustrative examples. Section 5 contains some concluding remarks, where we give another perturbation series and compare its corresponding results with ones by (1.12).

\section{Hereditary, Hamiltonian or symplectic operators by per- turbation}

We make a perturbation series for any $m \geq 0$

$$
\hat{u}_{m}=\sum_{i=0}^{m} \varepsilon^{i} \eta_{i}, \hat{\eta}_{m}=\left(\eta_{0}^{T}, \eta_{1}^{T}, \cdots, \eta_{m}^{T}\right)^{T},
$$

where $\eta_{i}, 0 \leq i \leq m$, are all column vectors possessing the same dimension as $u$. We first analyze a few of properties of tensor fields and then establish three useful theorems to construct hereditary, Hamiltonian or symplectic operators in terms of a perturbation. 
Lemma 2.1 We have for any $X \in T_{s}^{r}(M)$

$$
\frac{\partial^{k} X\left(\hat{u}_{i}\right)}{\partial \varepsilon^{k}}=\frac{\partial^{k} X\left(\hat{u}_{j}\right)}{\partial \varepsilon^{k}}, i, j \geq k \geq 0
$$

Proof: Let $j>i$ without loss of generality. Then we have

$$
\hat{u}_{j}=\hat{u}_{i}+\sum_{l=i+1}^{j} \varepsilon^{l} \eta_{l}
$$

Further we get

$$
X\left(\hat{u}_{j}\right)=X\left(\hat{u}_{i}\right)+\mathrm{o}\left(\varepsilon^{i}\right)
$$

from which the required equality follows. \#

Let us assume for any $X \in T_{s}^{r}(M)$

$$
(X(u))^{(i)}\left(\hat{\eta}_{j}\right)=\left.\frac{1}{i !} \frac{\partial^{i} X\left(\hat{u}_{j}\right)}{\partial \varepsilon^{i}}\right|_{\varepsilon=0}, X^{(i)}=(X(u))^{(i)}=(X(u))^{(i)}\left(\hat{\eta}_{i}\right), j \geq i \geq 0 .
$$

We write

$$
\left(\operatorname{per}_{m} X\right)\left(\hat{\eta}_{m}\right)=\hat{X}_{m}\left(\hat{\eta}_{m}\right)=\left(X^{(0) T}\left(\hat{\eta}_{m}\right), X^{(1) T}\left(\hat{\eta}_{m}\right), \cdots, X^{(m) T}\left(\hat{\eta}_{m}\right)\right)^{T}(m \geq 0)
$$

and call $\operatorname{per}_{m} X=\hat{X}_{m}$ the perturbation tensor field of order $m$. From Lemma 2.1, we see that

$$
\hat{X}_{m}\left(\hat{\eta}_{m}\right)=\left(X^{(0) T}\left(\hat{\eta}_{0}\right), X^{(1) T}\left(\hat{\eta}_{1}\right), \cdots, X^{(m) T}\left(\hat{\eta}_{m}\right)\right)^{T}
$$

and hence the perturbation tensor field $\operatorname{per}_{m} X=\hat{X}_{m}$ has a characteristic: the $i$-th component depends only on $\eta_{0}, \eta_{1}, \cdots, \eta_{i}$, not on any $\eta_{j}, j>i$.

Lemma 2.2 Let $X \in T_{s}^{r}(M)$ and $S \in T(M)$, we have

$$
\left(X^{\prime}(u)[S(u)]\right)^{(i)}\left(\hat{\eta}_{i}\right)=\left((X(u))^{(i)}\right)^{\prime}\left(\hat{\eta}_{i}\right)\left[\hat{S}_{i}\right], i \geq 0,
$$

where $\hat{S}_{i}=\left(S^{(0) T}, S^{(1) T}, \cdots, S^{(i) T}\right)^{T}$.

Proof: We first have

$$
\begin{aligned}
& \left(X\left(\hat{u}_{i}\right)\right)^{\prime}\left(\hat{\eta}_{i}\right)\left[\hat{S}_{i}\right]=\left.\frac{\partial}{\partial \delta}\right|_{\delta=0} X\left(\hat{u}_{i}+\delta \sum_{k=0}^{i} \varepsilon^{k} S^{(k)}\right) \\
= & \left.\frac{\partial}{\partial \delta}\right|_{\delta=0} K\left(\hat{u}_{i}+\delta S\left(\hat{u}_{i}\right)+\delta \mathrm{o}\left(\varepsilon^{i}\right)\right) \\
= & X^{\prime}\left(\hat{u}_{i}\right)\left[S\left(\hat{u}_{i}\right)\right]+\mathrm{o}\left(\varepsilon^{i}\right) .
\end{aligned}
$$


We apply the above equality to the following Taylor series

$$
X\left(\hat{u}_{i}\right)=\left.\sum_{k=0}^{i} \frac{\varepsilon^{k}}{i !} \frac{\partial^{k} X\left(\hat{u}_{i}\right)}{\partial \varepsilon^{k}}\right|_{\varepsilon=0}+\mathrm{o}\left(\varepsilon^{i}\right)
$$

and then get the required equality (2.5). \#

Evidently, (2.5) implies that for $X \in T_{s}^{r}(M), S \in T(M)$ we have

$$
\left(\operatorname{per}_{m}\left(X^{\prime}(u)[S(u)]\right)\right)\left(\hat{\eta}_{m}\right)=\left(\operatorname{per}_{m} X\left(\hat{\eta}_{m}\right)\right)^{\prime}\left(\hat{\eta}_{m}\right)\left[\left(\operatorname{per}_{m} S\right)\left(\hat{\eta}_{m}\right)\right] .
$$

Lemma 2.3 Let $X \in T_{s}^{r}(M)$. The following equalities hold for any vector field $\bar{S}_{N}=$ $\left(S_{0}^{T}, S_{1}^{T}, \cdots, S_{N}^{T}\right)^{T} \in T\left(\hat{M}_{N}\right)$, where $S_{i}, 0 \leq i \leq N$, are of the same dimension,

$$
\left(\left.\frac{\partial^{i} X\left(\hat{u}_{N}\right)}{\partial \varepsilon^{i}}\right|_{\varepsilon=0}\right)^{\prime}\left(\hat{\eta}_{N}\right)\left[\bar{S}_{N}\right]=\left.\frac{\partial^{i}}{\partial \varepsilon^{i}}\right|_{\varepsilon=0} X^{\prime}\left(\hat{u}_{N}\right)\left[\sum_{j=0}^{N} \varepsilon^{j} S_{j}\right], 0 \leq i \leq N .
$$

Proof: First from Taylor series

$$
X\left(\hat{u}_{N}\right)=\left.\sum_{i=0}^{N} \frac{\varepsilon^{i}}{i !} \frac{\partial^{i} X\left(\hat{u}_{N}\right)}{\partial \varepsilon^{i}}\right|_{\varepsilon=0}+\mathrm{o}\left(\varepsilon^{N}\right)
$$

we directly obtain

$$
\left(X\left(\hat{u}_{N}\right)\right)^{\prime}\left(\hat{\eta}_{N}\right)\left[\bar{S}_{N}\right]=\sum_{i=0}^{N} \frac{\varepsilon^{i}}{i !}\left(\left.\frac{\partial^{i} X\left(\hat{u}_{N}\right)}{\partial \varepsilon^{i}}\right|_{\varepsilon=0}\right)^{\prime}\left(\hat{\eta}_{N}\right)\left[\bar{S}_{N}\right]+\mathrm{o}\left(\varepsilon^{N}\right) .
$$

On the other hand, we have

$$
\left(X\left(\hat{u}_{N}\right)\right)^{\prime}\left(\hat{\eta}_{N}\right)\left[\bar{S}_{N}\right]=\left.\frac{\partial}{\partial \delta}\right|_{\delta=0} X\left(\hat{u}_{N}+\delta \sum_{j=0}^{N} \varepsilon^{j} S_{j}\right)=X^{\prime}\left(\hat{u}_{N}\right)\left[\sum_{j=0}^{N} \varepsilon^{j} S_{j}\right] .
$$

These two equalities give (2.6) again according to Taylor series. The proof is finished. \#

Theorem 2.1 If the operator $\Phi: T(M) \rightarrow T(M)$ is hereditary, then the operator $\hat{\Phi}_{N}$ : $T\left(\hat{M}_{N}\right) \rightarrow T\left(\hat{M}_{N}\right)$ defined by

$$
\begin{aligned}
& \left(\operatorname{per}_{N} \Phi\right)\left(\hat{\eta}_{N}\right)=\hat{\Phi}_{N}\left(\hat{\eta}_{N}\right) \\
= & {\left[\left(\hat{\Phi}_{N}\left(\hat{\eta}_{N}\right)\right)_{i j}\right]_{i, j=0,1, \cdots, N}=\left[\left.\frac{1}{(i-j) !} \frac{\partial^{i-j} \Phi\left(\hat{u}_{N}\right)}{\partial \varepsilon^{i-j}}\right|_{\varepsilon=0}\right]_{(N+1) \times(N+1)} } \\
= & {\left[\begin{array}{ccc}
\Phi\left(\eta_{0}\right) & 0 \\
\left.\frac{1}{1 !} \frac{\partial \Phi\left(\hat{u}_{N}\right)}{\partial \varepsilon}\right|_{\varepsilon=0} & \Phi\left(\eta_{0}\right) \\
\vdots & \ddots & \ddots \\
\left.\frac{1}{N !} \frac{\partial^{N} \Phi\left(\hat{u}_{N}\right)}{\partial \varepsilon^{N}}\right|_{\varepsilon=0} & \cdots & \left.\frac{1}{1 !} \frac{\partial \Phi\left(\hat{u}_{N}\right)}{\partial \varepsilon}\right|_{\varepsilon=0}
\end{array}\right] }
\end{aligned}
$$

is hereditary, too. 
Proof: Let $\bar{K}_{N}=\left(K_{0}^{T}, K_{1}^{T}, \cdots, K_{N}^{T}\right)^{T}, \bar{S}_{N}=\left(S_{0}^{T}, S_{1}^{T}, \cdots, S_{N}^{T}\right)^{T} \in T\left(\hat{M}_{N}\right)$, where $K_{i}, S_{i}, 0 \leq$ $i \leq N$, are of the same dimension. It suffices to prove that

$$
\begin{aligned}
& \hat{\Phi}_{N}^{\prime}\left(\hat{\eta}_{N}\right)\left[\hat{\Phi}_{N} \bar{K}_{N}\right] \bar{S}_{N}-\hat{\Phi}_{N} \hat{\Phi}_{N}^{\prime}\left(\hat{\eta}_{N}\right)\left[\bar{K}_{N}\right] \bar{S}_{N} \\
& -\hat{\Phi}_{N}^{\prime}\left(\hat{\eta}_{N}\right)\left[\hat{\Phi}_{N} \bar{S}_{N}\right] \bar{K}_{N}+\hat{\Phi}_{N} \hat{\Phi}_{N}^{\prime}\left(\hat{\eta}_{N}\right)\left[\bar{S}_{N}\right] \bar{K}_{N}=0 .
\end{aligned}
$$

First we easily get the $i$-th element of the vector field $\hat{\Phi}_{N} \bar{K}_{N}$ and the element in the $(i, j)$ position of the matrix $\hat{\Phi}_{N}^{\prime}\left(\hat{\eta}_{N}\right)\left[\bar{K}_{N}\right]$ :

$$
\begin{gathered}
\left(\hat{\Phi}_{N} \bar{K}_{N}\right)_{i}=\left.\sum_{j=0}^{i} \frac{1}{(i-j) !} \frac{\partial^{i-j} \Phi\left(\hat{u}_{N}\right)}{\partial \varepsilon^{i-j}}\right|_{\varepsilon=0} K_{j}, 0 \leq i \leq N, \\
\left(\hat{\Phi}_{N}^{\prime}\left(\hat{\eta}_{N}\right)\left[\bar{K}_{N}\right]\right)_{i j}=\frac{1}{(i-j) !}\left(\left.\frac{\partial^{i-j} \Phi\left(\hat{u}_{N}\right)}{\partial \varepsilon^{i-j}}\right|_{\varepsilon=0}\right)^{\prime}\left(\hat{\eta}_{N}\right)\left[\bar{K}_{N}\right] \\
=\left.\frac{1}{(i-j) !} \frac{\partial^{i-j}}{\partial \varepsilon^{i-j}}\right|_{\varepsilon=0}\left(\Phi^{\prime}\left(\hat{u}_{N}\right)\left[\sum_{k=0}^{N} \varepsilon^{k} K_{k}\right]\right), 0 \leq i, j \leq N .
\end{gathered}
$$

Here we use Lemma 2.3 for the calculation of the second equality. Now we can compute the $i$-th element of $\hat{\Phi}_{N}^{\prime}\left(\hat{\eta}_{N}\right)\left[\hat{\Phi}_{N} \bar{K}_{N}\right] \bar{S}_{N}$ :

$$
\begin{aligned}
& \left(\hat{\Phi}_{N}^{\prime}\left(\hat{\eta}_{N}\right)\left[\hat{\Phi}_{N} \bar{K}_{N}\right] \bar{S}_{N}\right)_{i} \\
= & \left.\sum_{j=0}^{i} \frac{1}{(i-j) !} \frac{\partial^{i-j}}{\partial \varepsilon^{i-j}}\right|_{\varepsilon=0} \Phi^{\prime}\left(\hat{u}_{N}\right)\left[\left.\sum_{k=0}^{N} \varepsilon^{k} \sum_{l=0}^{k} \frac{1}{(k-l) !} \frac{\partial^{k-l} \Phi\left(\hat{u}_{N}\right)}{\partial \varepsilon^{k-l}}\right|_{\varepsilon=0} K_{l}\right] S_{j} \\
= & \left.\sum_{j=0}^{i} \frac{1}{(i-j) !} \frac{\partial^{i-j}}{\partial \varepsilon^{i-j}}\right|_{\varepsilon=0} \Phi^{\prime}\left(\hat{u}_{N}\right)\left[\left.\sum_{l=0}^{N} \varepsilon^{l} \sum_{k=l}^{N} \frac{\varepsilon^{k-l}}{(k-l) !} \frac{\partial^{k-l} \Phi\left(\hat{u}_{N}\right)}{\partial \varepsilon^{k-l}}\right|_{\varepsilon=0} K_{l}\right] S_{j} \\
= & \left.\sum_{j=0}^{i} \frac{1}{(i-j) !} \frac{\partial^{i-j}}{\partial \varepsilon^{i-j}}\right|_{\varepsilon=0} \Phi^{\prime}\left(\hat{u}_{N}\right)\left[\sum_{l=0}^{N} \varepsilon^{l}\left(\Phi\left(\hat{u}_{N}\right)+o\left(\varepsilon^{N-l}\right)\right) K_{l}\right] S_{j} \\
= & \left.\sum_{j=0}^{i} \frac{1}{(i-j) !} \frac{\partial^{i-j}}{\partial \varepsilon^{i-j}}\right|_{\varepsilon=0} \Phi^{\prime}\left(\hat{u}_{N}\right)\left[\sum_{l=0}^{N} \varepsilon^{l}\left(\Phi\left(\hat{u}_{N}\right)\right) K_{l}\right] S_{j} \\
= & \left.\sum_{0 \leq j+l \leq i} \frac{1}{(i-j-l) !} \frac{\partial^{i-j-l}}{\partial \varepsilon^{i-j-l}}\right|_{\varepsilon=0} \Phi^{\prime}\left(\hat{u}_{N}\right)\left[\Phi\left(\hat{u}_{N}\right) K_{l}\right] S_{j}, 0 \leq i \leq N,
\end{aligned}
$$

and the $i$-th element of $\hat{\Phi}_{N} \hat{\Phi}_{N}^{\prime}\left(\hat{\eta}_{N}\right)\left[\bar{K}_{N}\right] \bar{S}_{N}$ :

$$
\begin{aligned}
& \left(\hat{\Phi}_{N} \hat{\Phi}_{N}^{\prime}\left(\hat{\eta}_{N}\right)\left[\bar{K}_{N}\right] \bar{S}_{N}\right)_{i} \\
= & \left.\left.\sum_{j=0}^{i} \frac{1}{(i-j) !} \frac{\partial^{i-j} \Phi\left(\hat{u}_{N}\right)}{\partial \varepsilon^{i-j}}\right|_{\varepsilon=0} \sum_{k=0}^{j} \frac{1}{(j-k) !} \frac{\partial^{j-k}}{\partial \varepsilon^{j-k}}\right|_{\varepsilon=0} \Phi^{\prime}\left(\hat{u}_{N}\right)\left[\sum_{l=0}^{N} \varepsilon^{l} K_{l}\right] S_{k} \\
= & \left.\left.\sum_{j=0}^{i} \frac{1}{(i-j) !} \frac{\partial^{i-j} \Phi\left(\hat{u}_{N}\right)}{\partial \varepsilon^{i-j}}\right|_{\varepsilon=0} \sum_{k=0}^{j} \sum_{l=0}^{j-k} \frac{1}{(j-k-l) !} \frac{\partial^{j-k-l}}{\partial \varepsilon^{j-k-l}}\right|_{\varepsilon=0} \Phi^{\prime}\left(\hat{u}_{N}\right)\left[K_{l}\right] S_{k}
\end{aligned}
$$




$$
\begin{aligned}
& =\left.\left.\sum_{k=0}^{i} \sum_{j=k}^{i} \sum_{l=0}^{j-k} \frac{1}{(i-j) !(j-k-l) !} \frac{\partial^{i-j} \Phi\left(\hat{u}_{N}\right)}{\partial \varepsilon^{i-j}}\right|_{\varepsilon=0} \frac{\partial^{j-k-l}}{\partial \varepsilon^{j-k-l}}\right|_{\varepsilon=0} \Phi^{\prime}\left(\hat{u}_{N}\right)\left[K_{l}\right] S_{k} \\
& =\left.\left.\sum_{k=0}^{i} \sum_{l=0}^{i-k} \sum_{j=k+l}^{i} \frac{1}{(i-j) !(j-k-l) !} \frac{\partial^{i-j} \Phi\left(\hat{u}_{N}\right)}{\partial \varepsilon^{i-j}}\right|_{\varepsilon=0} \frac{\partial^{j-k-l}}{\partial \varepsilon^{j-k-l}}\right|_{\varepsilon=0} \Phi^{\prime}\left(\hat{u}_{N}\right)\left[K_{l}\right] S_{k} \\
& =\left.\sum_{k=0}^{i} \sum_{l=0}^{i-k} \frac{1}{(i-k-l) !} \frac{\partial^{i-k-l}}{\partial \varepsilon^{i-k-l}}\right|_{\varepsilon=0}\left(\Phi\left(\hat{u}_{N}\right) \Phi^{\prime}\left(\hat{u}_{N}\right)\left[K_{l}\right] S_{k}\right) \\
& =\left.\sum_{0 \leq k+l \leq i} \frac{1}{(i-k-l) !} \frac{\partial^{i-k-l}}{\partial \varepsilon^{i-k-l}}\right|_{\varepsilon=0}\left(\Phi\left(\hat{u}_{N}\right) \Phi^{\prime}\left(\hat{u}_{N}\right)\left[K_{l}\right] S_{k}\right), 0 \leq i \leq N .
\end{aligned}
$$

Therefore by the hereditary property of $\Phi$, we find that each element in the left side of (2.8) is zero, which shows (2.8) holds. The proof is completed. \#

Theorem 2.2 If the operator $J: T^{*}(M) \rightarrow T(M)$ is Hamiltonian, then the operator $\hat{J}_{N}: T^{*}\left(\hat{M}_{N}\right) \rightarrow T\left(\hat{M}_{N}\right)$ defined by

$$
\begin{aligned}
& \left(\operatorname{per}_{N} J\right)\left(\hat{\eta}_{N}\right)=\hat{J}_{N}\left(\hat{\eta}_{N}\right) \\
& =\left[\left(\hat{J}_{N}\left(\hat{\eta}_{N}\right)\right)_{i j}\right]_{i, j=0,1, \cdots, N}=\left[\left.\frac{1}{(i+j-N) !} \frac{\partial^{i+j-N} J\left(\hat{u}_{N}\right)}{\partial \varepsilon^{i+j-N}}\right|_{\varepsilon=0}\right]_{(N+1) \times(N+1)} \\
& =\left[\begin{array}{cccc}
0 & & & J\left(\eta_{0}\right) \\
& & \vdots & \left.\frac{1}{1 !} \frac{\partial J\left(\hat{u}_{N}\right)}{\partial \varepsilon}\right|_{\varepsilon=0} \\
& \vdots & \vdots & \vdots \\
J\left(\eta_{0}\right) & \left.\frac{1}{1 !} \frac{\partial J\left(\hat{u}_{N}\right)}{\partial \varepsilon}\right|_{\varepsilon=0} & \cdots & \left.\frac{1}{N !} \frac{\partial^{N} J\left(\hat{u}_{N}\right)}{\partial \varepsilon^{N}}\right|_{\varepsilon=0}
\end{array}\right]
\end{aligned}
$$

is Hamiltonian, too.

Proof: Let $\bar{\alpha}_{N}=\left(\alpha_{0}^{T}, \alpha_{1}^{T}, \cdots, \alpha_{N}^{T}\right)^{T}, \bar{\beta}_{N}=\left(\beta_{0}^{T}, \beta_{1}^{T}, \cdots, \beta_{N}^{T}\right)^{T}, \bar{\gamma}_{N}=\left(\gamma_{0}^{T}, \gamma_{1}^{T}, \cdots, \gamma_{N}^{T}\right)^{T} \in$ $T^{*}\left(\hat{M}_{N}\right)$, where $\alpha_{i}, \beta_{i}, \gamma_{i} 0 \leq i \leq N$, are of the same dimension. We only need to prove that

$$
<\bar{\alpha}_{N}, \hat{J}_{N}^{\prime}\left(\hat{\eta}_{N}\right)\left[\hat{J}_{N} \bar{\beta}_{N}\right] \bar{\gamma}_{N}>+\operatorname{cycle}\left(\bar{\alpha}_{N}, \bar{\beta}_{N}, \bar{\gamma}_{N}\right)=0
$$

because there doesn't exist any problem on linearity and the skew-symmetric property. First noting Lemma 2.3, we can compute the element in the $(i, j)$ position of the matrix $\hat{J}_{N}^{\prime}\left(\hat{\eta}_{N}\right)\left[\hat{J}_{N} \bar{\beta}_{N}\right]$ :

$$
\begin{aligned}
& \left(\hat{J}_{N}^{\prime}\left(\hat{\eta}_{N}\right)\left[\hat{J}_{N} \bar{\beta}_{N}\right]\right)_{i j} \\
= & \left.\frac{1}{(i+j-N) !} \frac{\partial^{i+j-N}}{\partial \varepsilon^{i+j-N}}\right|_{\varepsilon=0} J^{\prime}\left(\hat{u}_{N}\right)\left[\sum_{l=0}^{N} \varepsilon^{l}\left(\hat{J}_{N} \bar{\beta}_{N}\right)_{l}\right] \\
= & \left.\frac{1}{(i+j-N) !} \frac{\partial^{i+j-N}}{\partial \varepsilon^{i+j-N}}\right|_{\varepsilon=0} J^{\prime}\left(\hat{u}_{N}\right)\left[\left.\sum_{l=0}^{N} \varepsilon^{l} \sum_{k=N-l}^{N} \frac{1}{(k+l-N) !} \frac{\partial^{k+l-N} J\left(\hat{u}_{N}\right)}{\partial \varepsilon^{k+l-N}}\right|_{\varepsilon=0} \beta_{k}\right]
\end{aligned}
$$




$$
\begin{aligned}
& =\left.\frac{1}{(i+j-N) !} \frac{\partial^{i+j-N}}{\partial \varepsilon^{i+j-N}}\right|_{\varepsilon=0} J^{\prime}\left(\hat{u}_{N}\right)\left[\sum_{k=0}^{N} \varepsilon^{N-k}\left(\left.\sum_{l=N-k}^{N} \frac{\varepsilon^{k+l-N}}{(k+l-N) !} \frac{\partial^{k+l-N} J\left(\hat{u}_{N}\right)}{\partial \varepsilon^{k+l-N}}\right|_{\varepsilon=0}\right) \beta_{k}\right] \\
& =\left.\frac{1}{(i+j-N) !} \frac{\partial^{i+j-N}}{\partial \varepsilon^{i+j-N}}\right|_{\varepsilon=0} J^{\prime}\left(\hat{u}_{N}\right)\left[\sum_{k=0}^{N} \varepsilon^{N-k}\left(J\left(\hat{u}_{N}\right)+\mathrm{o}\left(\varepsilon^{k}\right)\right) \beta_{k}\right] \\
& =\left.\frac{1}{(i+j-N) !} \frac{\partial^{i+j-N}}{\partial \varepsilon^{i+j-N}}\right|_{\varepsilon=0} J^{\prime}\left(\hat{u}_{N}\right)\left[\sum_{k=0}^{N} \varepsilon^{N-k} J\left(\hat{u}_{N}\right) \beta_{k}\right] \\
& =\left.\sum_{k=0}^{N} \frac{1}{(i+j-N) !} \frac{\partial^{i+j-N}}{\partial \varepsilon^{i+j-N}}\right|_{\varepsilon=0}\left(\varepsilon^{N-k} J^{\prime}\left(\hat{u}_{N}\right)\left[J\left(\hat{u}_{N}\right) \beta_{k}\right]\right) \\
& =\left.\sum_{k=2 N-(i+j)}^{N} \frac{1}{(i+j+k-2 N) !} \frac{\partial^{i+j+k-2 N}}{\partial \varepsilon^{i+j+k-2 N}}\right|_{\varepsilon=0}\left(J^{\prime}\left(\hat{u}_{N}\right)\left[J\left(\hat{u}_{N}\right) \beta_{k}\right]\right), 0 \leq i, j \leq N .
\end{aligned}
$$

Therefore we have

$$
\begin{aligned}
& <\bar{\alpha}_{N}, \hat{J}_{N}^{\prime}\left(\hat{\eta}_{N}\right)\left[\hat{J}_{N} \bar{\beta}_{N}\right] \bar{\gamma}_{N}>+\operatorname{cycle}\left(\bar{\alpha}_{N}, \bar{\beta}_{N}, \bar{\gamma}_{N}\right) \\
& =\left.\sum_{2 N \leq i+j+k \leq 3 N} \frac{1}{(i+j+k-2 N) !} \frac{\partial^{i+j+k-2 N}}{\partial \varepsilon^{i+j+k-2 N}}\right|_{\varepsilon=0}\left(<\alpha_{i}, J^{\prime}\left(\hat{u}_{N}\right)\left[J\left(\hat{u}_{N}\right) \beta_{k}\right] \gamma_{j}>\right. \\
& \left.\quad+\operatorname{cycle}\left(\alpha_{i}, \beta_{k}, \gamma_{j}\right)\right)=0 .
\end{aligned}
$$

In the last step, we have utilized the Hamiltonian property of $J(u)$. So the required result is proved. \#

Completely similar to the above two theorems, we can show the following result.

Theorem 2.3 If the operator $\Theta: T(M) \rightarrow T^{*}(M)$ is symplectic, then the operator $\hat{\Theta}_{N}$ : $T\left(\hat{M}_{N}\right) \rightarrow T^{*}\left(\hat{M}_{N}\right)$ defined by

$$
\begin{aligned}
& \left(\operatorname{per}_{N} \Theta\right)\left(\hat{\eta}_{N}\right)=\hat{\Theta}_{N}\left(\hat{\eta}_{N}\right) \\
& =\left[\left(\hat{\Theta}_{N}\left(\hat{\eta}_{N}\right)\right)_{i j}\right]_{i, j=0,1, \cdots, N}=\left[\left.\frac{1}{(N-i-j) !} \frac{\partial^{N-i-j} \Theta\left(\hat{u}_{N}\right)}{\partial \varepsilon^{N-i-j}}\right|_{\varepsilon=0}\right]_{(N+1) \times(N+1)} \\
& =\left[\begin{array}{cccc}
\left.\frac{1}{N !} \frac{\partial^{N} \Theta\left(\hat{u}_{N}\right)}{\partial \varepsilon^{N}}\right|_{\varepsilon=0} & \cdots & \left.\frac{1}{1 !} \frac{\partial \Theta\left(\hat{u}_{N}\right)}{\partial \varepsilon}\right|_{\varepsilon=0} & \Theta\left(\eta_{0}\right) \\
\vdots & \vdots & \vdots & \\
\left.\frac{1}{1 !} \frac{\partial^{N} \Theta\left(\hat{u}_{N}\right)}{\partial \varepsilon^{N}}\right|_{\varepsilon=0} & \vdots & & \\
\Theta\left(\eta_{0}\right) & & & 0
\end{array}\right]
\end{aligned}
$$

is symplectic, too.

We mention that when $J$ and $\Theta$ are invertible and $J=\Theta^{-1}$, we have

$$
\left(\operatorname{per}_{N} J\right)\left(\hat{\eta}_{N}\right)=\left(\left(\operatorname{per}_{N} \Theta\right)\left(\hat{\eta}_{N}\right)\right)^{-1}
$$


which shows that the inverse of the perturbation symplectic operator $\left(\operatorname{per}_{N} \Theta\right)\left(\hat{\eta}_{N}\right)$ is Hamiltonian and vice versa. Note that the above new operators have a vector $\hat{\eta}_{N}$ of dependent variables and hence involve $q(N+1)$ dependent variables when $u$ is a $q$-dimensional vector. The above three theorems also provide us a method to generate new Hereditary, Hamiltonian or symplectic operators from a known one. This is interesting in the soliton theory. In particular, we can put forward the following operators by the first order perturbation:

$$
\begin{aligned}
& \left(\hat{\Phi}_{1}\right)\left(\hat{\eta}_{1}\right)=\left[\begin{array}{cc}
\Phi\left(\eta_{0}\right) & 0 \\
\Phi^{\prime}\left(\eta_{0}\right)\left[\eta_{1}\right] & \Phi\left(\eta_{0}\right)
\end{array}\right], \\
& \left(\hat{J}_{1}\right)\left(\hat{\eta}_{1}\right)=\left[\begin{array}{cc}
0 & J\left(\eta_{0}\right) \\
J\left(\eta_{0}\right) & J^{\prime}\left(\eta_{0}\right)\left[\eta_{1}\right]
\end{array}\right], \\
& \left(\hat{\Theta}_{1}\right)\left(\hat{\eta}_{1}\right)=\left[\begin{array}{cc}
\Theta^{\prime}\left(\eta_{0}\right)\left[\eta_{1}\right] & \Theta\left(\eta_{0}\right) \\
\Theta\left(\eta_{0}\right) & 0
\end{array}\right] .
\end{aligned}
$$

Taking the first order perturbation once more, we can obtain a little more complicated operators:

$$
\begin{aligned}
& \left(\operatorname{per}_{1} \operatorname{per}_{1} \Phi\right)\left(\hat{\eta}_{3}\right)=\left[\begin{array}{cccc}
\Phi\left(\eta_{0}\right) & 0 & 0 & 0 \\
\Phi^{\prime}\left(\eta_{0}\right)\left[\eta_{1}\right] & \Phi\left(\eta_{0}\right) & 0 & 0 \\
\Phi^{\prime}\left(\eta_{0}\right)\left[\eta_{2}\right] & 0 & \Phi^{\prime}\left(\eta_{0}\right)\left[\eta_{1}\right] & \Phi\left(\eta_{0}\right) \\
\left.\frac{\partial^{2} \Phi\left(\eta_{0}+\varepsilon \eta_{1}+\delta \eta_{2}+\varepsilon \delta \eta_{3}\right)}{\partial \varepsilon \partial \delta}\right|_{\varepsilon=\delta=0} & \Phi^{\prime}\left(\eta_{0}\right)\left[\eta_{2}\right] & \Phi^{\prime}\left(\eta_{0}\right)\left[\eta_{1}\right] & \Phi\left(\eta_{0}\right)
\end{array}\right], \\
& \left(\operatorname{per}_{1} \operatorname{per}_{1} J\right)\left(\hat{\eta}_{3}\right)=\left[\begin{array}{cccc}
0 & 0 & 0 & J\left(\eta_{0}\right) \\
0 & 0 & J\left(\eta_{0}\right) & J^{\prime}\left(\eta_{0}\right)\left[\eta_{1}\right] \\
0 & J\left(\eta_{0}\right) & 0 & J^{\prime}\left(\eta_{0}\right)\left[\eta_{2}\right] \\
J\left(\eta_{0}\right) & J^{\prime}\left(\eta_{0}\right)\left[\eta_{1}\right] & J^{\prime}\left(\eta_{0}\right)\left[\eta_{2}\right] & \left.\frac{\partial^{2} J\left(\eta_{0}+\varepsilon \eta_{1}+\delta \eta_{2}+\varepsilon \delta \eta_{3}\right)}{\partial \varepsilon \partial \delta}\right|_{\varepsilon=\delta=0}
\end{array}\right], \\
& \left(\operatorname{per}_{1} \operatorname{per}_{1} \Theta\right)\left(\hat{\eta}_{3}\right)=\left[\begin{array}{cccc}
\left.\frac{\partial^{2} \Theta\left(\eta_{0}+\varepsilon \eta_{1}+\delta \eta_{2}+\varepsilon \delta \eta_{3}\right)}{\partial \varepsilon \partial \delta}\right|_{\varepsilon=\delta=0} & \Theta^{\prime}\left(\eta_{0}\right)\left[\eta_{2}\right] & \Theta^{\prime}\left(\eta_{0}\right)\left[\eta_{1}\right] & \Theta\left(\eta_{0}\right) \\
\Theta^{\prime}\left(\eta_{0}\right)\left[\eta_{2}\right] & 0 & \Theta\left(\eta_{0}\right) & 0 \\
\Theta^{\prime}\left(\eta_{0}\right)\left[\eta_{1}\right] & \Theta\left(\eta_{0}\right) & 0 & 0 \\
\Theta\left(\eta_{0}\right) & 0 & 0 & 0
\end{array}\right] .
\end{aligned}
$$

Here we have changed two dependent variables while making the second perturbation. Of course this kind of perturbation may be done without any stop at finite steps and hence the resulting operators are full of various algebraic structures.

\section{Integrable properties of the perturbation equations}

Let us recall the perturbation series (1.12)

$$
\hat{u}_{N}=\sum_{i=0}^{N} \varepsilon^{i} \eta_{i}, \hat{\eta}_{N}=\left(\eta_{0}^{T}, \eta_{1}^{T} \cdot \cdots, \eta_{N}^{T}\right)^{T}, \quad N \geq 1 .
$$


For the evolution equation (1.11)

$$
u_{t}=K(u), K \in T(M),
$$

we consider the following $N$-th order perturbation equation

$$
\hat{u}_{N t}=K\left(\hat{u}_{N}\right)+\mathrm{o}\left(\varepsilon^{N}\right) \text { or } \hat{u}_{N t}=K\left(\hat{u}_{N}\right) \quad\left(\bmod \varepsilon^{N}\right) \text {, }
$$

which leads to the following equivalent equation

$$
\hat{\eta}_{N t}=\hat{K}_{N}\left(\hat{\eta}_{N}\right), \text { namely } \eta_{i t}=\left.\frac{1}{i !} \frac{\partial K\left(\hat{u}_{N}\right)}{\partial \varepsilon^{i}}\right|_{\varepsilon=0}, 0 \leq i \leq N
$$

In this section, we would like to discuss integrable properties of the perturbation equation (3.2), which include recursion operators, $K$-symmetries (i.e. time independent symmetries), master symmetries, linear representations (Lax representation and zero curvature representation) and Hamiltonian structures etc.

Theorem 3.1 Let $K \in T(M)$. The operator $\hat{\Phi}_{N}: T\left(\hat{M}_{N}\right) \rightarrow T\left(\hat{M}_{N}\right)$ determined by (2.7) is a recursion operator of the perturbation equation $\hat{\eta}_{N t}=\hat{K}_{N}\left(\hat{\eta}_{N}\right)$ defined by (3.马) when $\Phi: T(M) \rightarrow T(M)$ is a recursion operator of $u_{t}=K(u)$. Therefore the perturbation equation $\hat{\eta}_{N t}=\hat{K}_{N}\left(\hat{\eta}_{N}\right)$ has a hereditary recursion operator $\hat{\Phi}_{N}\left(\hat{\eta}_{N}\right)$ once $u_{t}=K(u)$ has a hereditary recursion operator $\Phi(u)$.

Proof: Let $\bar{S}_{N}=\left(S_{0}^{T}, S_{1}^{T}, \cdots, S_{N}^{T}\right)^{T} \in T\left(\hat{M}_{N}\right)$, where $S_{i}, 0 \leq i \leq N$, are of the same dimension. By Lemma 2.3, we have

$$
\begin{aligned}
& \left(\left.\frac{\partial^{k} \Phi\left(\hat{u}_{N}\right)}{\partial \varepsilon^{k}}\right|_{\varepsilon=0}\right)^{\prime}\left(\hat{\eta}_{N}\right)\left[\hat{K}_{N}\right]=\left.\frac{\partial^{k}}{\partial \varepsilon^{k}}\right|_{\varepsilon=0} \Phi^{\prime}\left(\hat{u}_{N}\right)\left[\sum_{j=0}^{N} \varepsilon^{j} K^{(j)}\right] \\
= & \left.\frac{\partial^{k}}{\partial \varepsilon^{k}}\right|_{\varepsilon=0} \Phi^{\prime}\left(\hat{u}_{N}\right)\left[K\left(\hat{u}_{N}\right)+o\left(\varepsilon^{N}\right)\right]=\left.\frac{\partial^{k} \Phi^{\prime}\left(\hat{u}_{N}\right)\left[K\left(\hat{u}_{N}\right)\right]}{\partial \varepsilon^{k}}\right|_{\varepsilon=0}, 0 \leq k \leq N,
\end{aligned}
$$

and

$$
\begin{aligned}
& \left(K^{(i)}\right)^{\prime}\left(\hat{\eta}_{N}\right)\left[\bar{S}_{N}\right]=\left.\frac{1}{i !} \frac{\partial^{i}}{\partial \varepsilon^{i}}\right|_{\varepsilon=0} K^{\prime}\left(\hat{u}_{N}\right)\left[\sum_{k=0}^{N} \varepsilon^{k} S_{k}\right] \\
= & \left.\sum_{j=0}^{i} \frac{1}{(i-j) !} \frac{\partial^{i-j} K^{\prime}\left(\hat{u}_{N}\right)\left[S_{j}\right]}{\partial \varepsilon^{i-j}}\right|_{\varepsilon=0}, 0 \leq i \leq N .
\end{aligned}
$$

Therefore from the above first equality, we obtain the $i$-th element of $\hat{\Phi}_{N}^{\prime}\left(\hat{\eta}_{N}\right)\left[\hat{K}_{N}\right] \bar{S}_{N}$ :

$$
\left(\hat{\Phi}_{N}^{\prime}\left(\hat{\eta}_{N}\right)\left[\hat{K}_{N}\right] \bar{S}_{N}\right)_{i}=\left.\sum_{j=0}^{i} \frac{1}{(i-j) !} \frac{\partial^{i-j} \Phi^{\prime}\left(\hat{u}_{N}\right)\left[K\left(\hat{u}_{N}\right)\right] S_{j}}{\partial \varepsilon^{i-j}}\right|_{\varepsilon=0}, 0 \leq i \leq N
$$


From the above second equality, we can compute the following two terms:

$$
\begin{aligned}
& \left(\hat{K}_{N}^{\prime}\left(\hat{\eta}_{N}\right)\left[\hat{\Phi}_{N} \bar{S}_{N}\right]\right)_{i} \\
& =\left.\sum_{k=0}^{i} \frac{1}{(i-k) !} \frac{\partial^{i-k}}{\partial \varepsilon^{i-k}}\right|_{\varepsilon=0}\left(K^{\prime}\left(\hat{u}_{N}\right)\left[\left(\hat{\Phi}_{N} \bar{S}_{N}\right)_{k}\right]\right) \\
& =\left.\sum_{k=0}^{i} \frac{1}{(i-k) !} \frac{\partial^{i-k}}{\partial \varepsilon^{i-k}}\right|_{\varepsilon=0} K^{\prime}\left(\hat{u}_{N}\right)\left[\left.\sum_{j=0}^{k} \frac{1}{(k-j) !} \frac{\partial^{k-j} \Phi\left(\hat{u}_{N}\right)}{\partial \varepsilon^{k-j}}\right|_{\varepsilon=0} S_{j}\right] \\
& =\left.\sum_{j=0}^{i} \sum_{k=j}^{i} \frac{1}{(i-k) !(k-j) !} \frac{\partial^{i-k}}{\partial \varepsilon^{i-k}}\right|_{\varepsilon=0} K^{\prime}\left(\hat{u}_{N}\right)\left[\left.\frac{\partial^{k-j} \Phi\left(\hat{u}_{N}\right)}{\partial \varepsilon^{k-j}}\right|_{\varepsilon=0} S_{j}\right] \\
& =\left.\sum_{j=0}^{i} \sum_{k=j}^{i} \frac{1}{(i-j) !} \frac{\partial^{i-j}}{\partial \varepsilon^{i-j}}\right|_{\varepsilon=0}\left(\frac{\varepsilon^{k-j}}{(k-j) !} K^{\prime}\left(\hat{u}_{N}\right)\left[\left.\frac{\partial^{k-j} \Phi\left(\hat{u}_{N}\right)}{\partial \varepsilon^{k-j}}\right|_{\varepsilon=0} S_{j}\right]\right) \\
& =\left.\sum_{j=0}^{i} \frac{1}{(i-j) !} \frac{\partial^{i-j}}{\partial \varepsilon^{i-j}}\right|_{\varepsilon=0} K^{\prime}\left(\hat{u}_{N}\right)\left[\left.\sum_{k=j}^{i} \frac{\varepsilon^{k-j}}{(k-j) !} \frac{\partial^{k-j} \Phi\left(\hat{u}_{N}\right)}{\partial \varepsilon^{k-j}}\right|_{\varepsilon=0} S_{j}\right] \\
& =\left.\sum_{j=0}^{i} \frac{1}{(i-j) !} \frac{\partial^{i-j}}{\partial \varepsilon^{i-j}}\right|_{\varepsilon=0} K^{\prime}\left(\hat{u}_{N}\right)\left[\Phi\left(\hat{u}_{N}\right) S_{j}+\mathrm{o}\left(\varepsilon^{i-j}\right)\right] \\
& =\left.\sum_{j=0}^{i} \frac{1}{(i-j) !} \frac{\partial^{i-j}\left(K^{\prime}\left(\hat{u}_{N}\right)\left[\Phi\left(\hat{u}_{N}\right) S_{j}\right]\right)}{\partial \varepsilon^{i-j}}\right|_{\varepsilon=0}, 0 \leq i \leq N \text {; } \\
& \left(\hat{\Phi}_{N} \hat{K}_{N}^{\prime}\left(\hat{\eta}_{N}\right)\left[\bar{S}_{N}\right]\right)_{i} \\
& =\left.\left.\sum_{k=0}^{i} \frac{1}{(i-k) !} \frac{\partial^{i-k} \Phi\left(\hat{u}_{N}\right)}{\partial \varepsilon^{i-k}}\right|_{\varepsilon=0} \sum_{j=0}^{k} \frac{1}{(k-j) !} \frac{\partial^{k-j} K^{\prime}\left(\hat{u}_{N}\right)\left[S_{j}\right]}{\partial \varepsilon^{k-j}}\right|_{\varepsilon=0} \\
& =\left.\left.\sum_{j=0}^{i} \sum_{k=j}^{i} \frac{1}{(i-k) !(k-j) !} \frac{\partial^{i-k} \Phi\left(\hat{u}_{N}\right)}{\partial \varepsilon^{i-k}}\right|_{\varepsilon=0} \frac{\partial^{k-j} K^{\prime}\left(\hat{u}_{N}\right)\left[S_{j}\right]}{\partial \varepsilon^{k-j}}\right|_{\varepsilon=0} \\
& =\left.\sum_{j=0}^{i} \frac{1}{(i-j) !} \frac{\partial^{i-j}\left(\Phi\left(\hat{u}_{N}\right) K^{\prime}\left(\hat{u}_{N}\right)\left[S_{j}\right]\right)}{\partial \varepsilon^{i-k}}\right|_{\varepsilon=0}, 0 \leq i \leq N .
\end{aligned}
$$

Now in virtue of the above three equalities (3.3), (3.4) and (3.5), we easily see that

$$
\frac{\partial \hat{\Phi}_{N}}{\partial t} \bar{S}_{N}+\hat{\Phi}_{N}^{\prime}\left(\hat{\eta}_{N}\right)\left[\hat{K}_{N}\right] \bar{S}_{N}-\hat{K}_{N}^{\prime}\left(\hat{\eta}_{N}\right)\left[\hat{\Phi}_{N} \bar{S}_{N}\right]+\hat{\Phi}_{N} \hat{K}_{N}^{\prime}\left(\hat{\eta}_{N}\right)\left[\bar{S}_{N}\right]=0
$$

which implies that $\hat{\Phi}_{N}\left(\hat{\eta}_{N}\right)$ is a recursion operator of $\hat{\eta}_{N t}=\hat{K}_{N}$. A combination with Theorem 2.1 gives the proof of the second conclusion. \#

Theorem 3.2 Let $K, S \in T(M)$. There exists a relation between the perturbation vector fields $\hat{K}_{N}$ and $\hat{S}_{N}$

$$
\left[\hat{K}_{N}\left(\hat{\eta}_{N}\right), \hat{S}_{N}\left(\hat{\eta}_{N}\right)\right]=\left(\hat{K}_{N}\right)^{\prime}\left(\hat{\eta}_{N}\right)\left[\hat{S}_{N}\left(\hat{\eta}_{N}\right)\right]-\left(\hat{S}_{N}\right)^{\prime}\left(\hat{\eta}_{N}\right)\left[\hat{K}_{N}\left(\hat{\eta}_{N}\right)\right]=\hat{T}_{N}\left(\hat{\eta}_{N}\right)
$$


where $\hat{T}_{N}\left(\hat{\eta}_{N}\right)$ is the perturbation vector field of the vector field $T=[K, S] \in T(M)$. Therefore we have

(1) if $\sigma \in T(M)$ is an $n$-th order master-symmetry of the equation $u_{t}=K(u)$, then $\hat{\sigma}_{N}=$ $\left(\sigma^{(0) T}, \sigma^{(1) T}, \cdots, \sigma^{(N) T}\right)^{T} \in T\left(\hat{M}_{N}\right)$ is an $n$-th order master-symmetry of the perturbation equation $\hat{\eta}_{N t}=\hat{K}_{N}\left(\hat{\eta}_{N}\right)$;

(2) the perturbation equation $\hat{\eta}_{N t}=\hat{K}_{N}\left(\hat{\eta}_{N}\right)$ possesses the same symmetry algebra structure as the original equation $u_{t}=K(u)$.

Proof: By Lemma 2.2, we see for the $i$-th element that

$$
\begin{aligned}
& (T(u))^{(i)}=\left(K^{\prime}(u)[S(u)]\right)^{(i)}\left(\hat{\eta}_{i}\right)-\left(S^{\prime}(u)[K(u)]\right)^{(i)}\left(\hat{\eta}_{i}\right) \\
= & \left((K(u))^{(i)}\right)^{\prime}\left(\hat{\eta}_{i}\right)\left[\hat{S}_{i}\right]-\left((S(u))^{(i)}\right)^{\prime}\left(\hat{\eta}_{i}\right)\left[\hat{K}_{i}\right] \\
= & \left(\hat{K}_{N}^{\prime}\left(\hat{\eta}_{N}\right)\left[\hat{S}_{N}\right]\right)_{i}-\left(\hat{S}_{N}^{\prime}\left(\hat{\eta}_{N}\right)\left[\hat{K}_{N}\right]\right)_{i}, 0 \leq i \leq N,
\end{aligned}
$$

which shows (3.6) holds. The rest of the proof is obvious. The proof is finished. \#

The relation (3.6) implies that the perturbation series (1.12) keeps Lie product of vector fields invariant.

Theorem 3.3 Let $K \in T(M)$. When the evolution equation $u_{t}=K(u)$ has a Lax representation $(L(u))_{t}=[A(u), L(u)]$ where $L, A$ are two matrix differential operators, the $N$-th order perturbation equation $\hat{\eta}_{N t}=\hat{K}_{N}\left(\hat{\eta}_{N}\right)$ has the following Lax representation

$$
\left(\hat{L}_{N}\left(\hat{\eta}_{N}\right)\right)_{t}=\left[\hat{A}_{N}\left(\hat{\eta}_{N}\right), \hat{L}_{N}\left(\hat{\eta}_{N}\right)\right]
$$

where the spectral operator $\hat{L}_{N}$ and the Lax operator $\hat{A}_{N}$ read as

$$
\begin{aligned}
& \left(\operatorname{per}_{N} L\right)\left(\hat{\eta}_{N}\right)=\hat{L}_{N}\left(\hat{\eta}_{N}\right) \\
& =\left[\left(\hat{L}_{N}\left(\hat{\eta}_{N}\right)\right)_{i j}\right]_{i, j=0,1, \cdots, N}=\left[\left.\frac{1}{(i-j) !} \frac{\partial^{i-j} L\left(\hat{u}_{N}\right)}{\partial \varepsilon^{i-j}}\right|_{\varepsilon=0}\right]_{(N+1) \times(N+1)} \\
& =\left[\begin{array}{cccc}
L\left(\eta_{0}\right) & & & 0 \\
\left.\frac{1}{1 !} \frac{\partial L\left(\hat{u}_{N}\right)}{\partial \varepsilon}\right|_{\varepsilon=0} & L\left(\eta_{0}\right) & & \\
\vdots & \ddots & \ddots & \\
\left.\frac{1}{N !} \frac{\partial^{N} L\left(\hat{u}_{N}\right)}{\partial \varepsilon^{N}}\right|_{\varepsilon=0} & \cdots & \left.\frac{1}{1 !} \frac{\partial L\left(\hat{u}_{N}\right)}{\partial \varepsilon}\right|_{\varepsilon=0} & L\left(\eta_{0}\right)
\end{array}\right], \\
& \left(\operatorname{per}_{N} A\right)\left(\hat{\eta}_{N}\right)=\hat{A}_{N}\left(\hat{\eta}_{N}\right) \\
& =\left[\left(\hat{A}_{N}\left(\hat{\eta}_{N}\right)\right)_{i j}\right]_{i, j=0,1, \cdots, N}=\left[\left.\frac{1}{(i-j) !} \frac{\partial^{i-j} A\left(\hat{u}_{N}\right)}{\partial \varepsilon^{i-j}}\right|_{\varepsilon=0}\right]_{(N+1) \times(N+1)} \\
& =\left[\begin{array}{cccc}
A\left(\eta_{0}\right) & & & 0 \\
\left.\frac{1}{1 !} \frac{\partial A\left(\hat{u}_{N}\right)}{\partial \varepsilon}\right|_{\varepsilon=0} & A\left(\eta_{0}\right) & & \\
\vdots & \ddots & \ddots & \\
\left.\frac{1}{N !} \frac{\partial^{N} A\left(\hat{u}_{N}\right)}{\partial \varepsilon^{N}}\right|_{\varepsilon=0} & \cdots & \left.\frac{1}{1 !} \frac{\partial A\left(\hat{u}_{N}\right)}{\partial \varepsilon}\right|_{\varepsilon=0} & A\left(\eta_{0}\right)
\end{array}\right] .
\end{aligned}
$$


Proof: We first note that

$$
\hat{u}_{N t}=K\left(\hat{u}_{N}\right)+\mathrm{o}\left(\varepsilon^{N}\right)
$$

and thus we have

$$
\left.\frac{\partial^{k}}{\partial \varepsilon^{k}}\right|_{\varepsilon=0}\left(\left(L\left(\hat{u}_{N}\right)\right)_{t}-\left[A\left(\hat{u}_{N}\right), L\left(\hat{u}_{N}\right)\right]\right)=0,0 \leq k \leq N .
$$

Let now us compute the elements of the differential operator matrix $\left[\hat{A}_{N}, \hat{L}_{N}\right]$. Evidently, we know that $\left[\hat{A}_{N}, \hat{L}_{N}\right]$ is lower-triangular, that is,

$$
\left(\left[\hat{A}_{N}, \hat{L}_{N}\right]\right)_{i j}=0,0 \leq i<j \leq N .
$$

On the other hand, when $0 \leq j \leq i \leq N$, we can compute

$$
\begin{aligned}
\left(\hat{A}_{N} \hat{L}_{N}\right)_{i j} & =\left.\left.\sum_{k=j}^{i} \frac{1}{(i-k) !} \frac{\partial^{i-k} A\left(\hat{u}_{N}\right)}{\partial \varepsilon^{i-k}}\right|_{\varepsilon=0} \frac{1}{(k-j) !} \frac{\partial^{k-j} L\left(\hat{u}_{N}\right)}{\partial \varepsilon^{k-j}}\right|_{\varepsilon=0} \\
& =\left.\left.\frac{1}{(i-j) !} \sum_{k=j}^{i}\left(\begin{array}{c}
i-j \\
i-k
\end{array}\right) \frac{\partial^{i-k} A\left(\hat{u}_{N}\right)}{\partial \varepsilon^{i-k}}\right|_{\varepsilon=0} \frac{\partial^{k-j} L\left(\hat{u}_{N}\right)}{\partial \varepsilon^{k-j}}\right|_{\varepsilon=0} \\
& =\left.\frac{1}{(i-j) !} \frac{\partial^{i-j} A\left(\hat{u}_{N}\right) L\left(\hat{u}_{N}\right)}{\partial \varepsilon^{i-j}}\right|_{\varepsilon=0} .
\end{aligned}
$$

In the same way, we obtain

$$
\left(\hat{L}_{N} \hat{A}_{N}\right)_{i j}=\left.\frac{1}{(i-j) !} \frac{\partial^{i-j} L\left(\hat{u}_{N}\right) A\left(\hat{u}_{N}\right)}{\partial \varepsilon^{i-j}}\right|_{\varepsilon=0} .
$$

Therefore we have

$$
\left(\left[\hat{A}_{N}, \hat{L}_{N}\right]\right)_{i j}=\left.\frac{1}{(i-j) !} \frac{\partial^{i-j}\left[A\left(\hat{u}_{N}\right), L\left(\hat{u}_{N}\right)\right]}{\partial \varepsilon^{i-j}}\right|_{\varepsilon=0}, 0 \leq i, j \leq N .
$$

Now we easily find that (3.7) is true due to (3.10). The proof is finished. \#

Note that the spectral operator $\hat{L}_{N}$ and the hereditary recursion operator $\hat{\Phi}_{N}$ have the same form of matrix. In fact, we can take the hereditary recursion operators as the spectral ones. More precisely, $u_{t}=K(u)$ has a Lax representation 24] $\Phi_{t}=\left[\Phi, K^{\prime}\right]$ where $\Phi$ and $K^{\prime}$ are a recursion operator and the Gateaux derivative operator of $K$, respectively. The following result for the case of zero curvature representation may also be shown. Its proof is omitted due to the completely similar deduction.

Theorem 3.4 Let $K \in T(M)$. When the evolution equation $u_{t}=K(u)$ has a zero curvature representation $(U(u))_{t}-(V(u))_{x}+[U(u), V(u)]=0$ where $U$ and $V$ are two matrix differential (sometimes multiplication) operators, the $N$-th order perturbation equation $\hat{\eta}_{N t}=\hat{K}_{N}\left(\hat{\eta}_{N}\right)$ has the following zero curvature representation

$$
\left(\hat{U}_{N}\left(\hat{\eta}_{N}\right)\right)_{t}-\left(\hat{U}_{N}\left(\hat{\eta}_{N}\right)\right)_{x}+\left[\hat{U}_{N}\left(\hat{\eta}_{N}\right), \hat{V}_{N}\left(\hat{\eta}_{N}\right)\right]=0
$$


where the two matrix differential operators $\hat{U}_{N}$ and $\hat{V}_{N}$ are of the form

$$
\begin{aligned}
& \left(\operatorname{per}_{N} U\right)\left(\hat{\eta}_{N}\right)=\hat{U}_{N}\left(\hat{\eta}_{N}\right) \\
& =\left[\left(\hat{U}_{N}\left(\hat{\eta}_{N}\right)\right)_{i j}\right]_{i, j=0,1, \cdots, N}=\left[\left.\frac{1}{(i-j) !} \frac{\partial^{i-j} U\left(\hat{u}_{N}\right)}{\partial \varepsilon^{i-j}}\right|_{\varepsilon=0}\right]_{(N+1) \times(N+1)}
\end{aligned}
$$

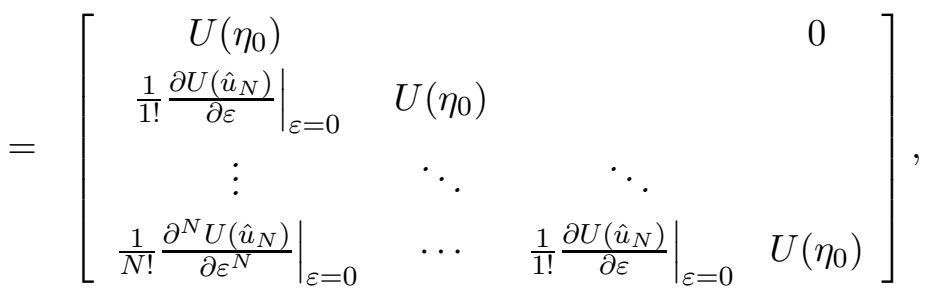

$$
\begin{aligned}
& \left(\operatorname{per}_{N} V\right)\left(\hat{\eta}_{N}\right)=\hat{V}_{N}\left(\hat{\eta}_{N}\right) \\
& =\left[\left(\hat{V}_{N}\left(\hat{\eta}_{N}\right)\right)_{i j}\right]_{i, j=0,1, \cdots, N}=\left[\left.\frac{1}{(i-j) !} \frac{\partial^{i-j} V\left(\hat{u}_{N}\right)}{\partial \varepsilon^{i-j}}\right|_{\varepsilon=0}\right]_{(N+1) \times(N+1)}
\end{aligned}
$$

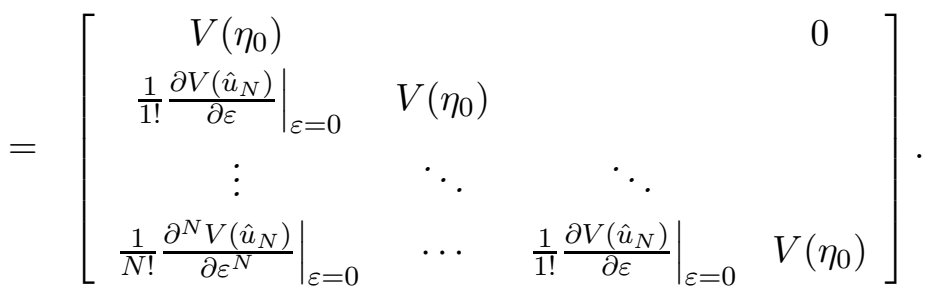

Theorem 3.5 Let $K \in T(M)$. If the equation $u_{t}=K(u)$ possesses a Hamiltonian structure

$$
u_{t}=K(u)=J(u) \frac{\delta H(u)}{\delta u}
$$

where $J: T^{*}(M) \rightarrow T(M)$ is a Hamiltonian operator and $H \in C^{\infty}(M)$ is a Hamiltonian function, then the perturbation equation $\hat{\eta}_{N t}=\hat{K}_{N}\left(\hat{\eta}_{N}\right)$ also possesses a Hamiltonian structure

$$
\hat{\eta}_{N t}=\hat{K}_{N}\left(\hat{\eta}_{N}\right)=\hat{J}_{N}\left(\hat{\eta}_{N}\right) \frac{\delta \hat{H}_{N}\left(\hat{\eta}_{N}\right)}{\delta \hat{\eta}_{N}}
$$

where the Hamiltonian operator $\hat{J}_{N}\left(\hat{\eta}_{N}\right)$ is given by (2.9) and the Hamiltonian function $\hat{H}_{N} \in C^{\infty}\left(\hat{M}_{N}\right)$ is determined by

$$
\left(\operatorname{per}_{N} H\right)\left(\hat{\eta}_{N}\right)=\hat{H}_{N}\left(\hat{\eta}_{N}\right)=\left.\frac{1}{N !} \frac{\partial^{N} H\left(\hat{u}_{N}\right)}{\partial \varepsilon^{N}}\right|_{\varepsilon=0} .
$$

The corresponding Poisson bracket has the property

$$
\left\{\hat{H}_{1 N}, \hat{H}_{2 N}\right\}_{\hat{J}_{N}}\left(\hat{\eta}_{N}\right)=\left.\frac{1}{N !} \frac{\partial^{N}}{\partial \varepsilon^{N}}\right|_{\varepsilon=0}\left\{H_{1}, H_{2}\right\}_{J}\left(\hat{u}_{N}\right), H_{1}, H_{2} \in C^{\infty}(M) .
$$


Furthermore the perturbation equation $\hat{\eta}_{N t}=\hat{K}_{N}\left(\hat{\eta}_{N}\right)$ possesses a multi-Hamiltonian structure

$$
\hat{\eta}_{N t}=\hat{K}_{N}\left(\hat{\eta}_{N}\right)=\hat{J}_{1 N}\left(\hat{\eta}_{N}\right) \frac{\delta \hat{H}_{1 N}\left(\hat{\eta}_{N}\right)}{\delta \hat{\eta}_{N}}=\cdots=\hat{J}_{m N}\left(\hat{\eta}_{N}\right) \frac{\delta \hat{H}_{m N}\left(\hat{\eta}_{N}\right)}{\delta \hat{\eta}_{N}}
$$

once $u_{t}=K(u)$ possesses an analogous multi-Hamiltonian structure

$$
u_{t}=K(u)=J_{1}(u) \frac{\delta H_{1}(u)}{\delta u}=\cdots=J_{m}(u) \frac{\delta H_{m}(u)}{\delta u} .
$$

Proof: Let $\gamma=\frac{\delta H}{\delta u} \in T^{*}(M)$. Then we have

$$
\begin{gathered}
\eta_{i t}=\left.\frac{1}{i !} \frac{\partial^{i} J\left(\hat{u}_{N}\right) \gamma\left(\hat{u}_{N}\right)}{\partial \varepsilon^{i}}\right|_{\varepsilon=0} \\
=\left.\left.\sum_{j=0}^{i} \frac{1}{j !(i-j) !} \frac{\partial^{i-j} J\left(\hat{u}_{N}\right)}{\partial \varepsilon^{i-j}}\right|_{\varepsilon=0} \frac{\partial^{j} \gamma\left(\hat{u}_{N}\right)}{\partial \varepsilon^{j}}\right|_{\varepsilon=0}, 0 \leq i \leq N .
\end{gathered}
$$

Thus we get

$$
\hat{\eta}_{N t}=\hat{K}_{N}\left(\hat{\eta}_{N}\right)=\hat{J}_{N}\left(\hat{\eta}_{N}\right) \hat{\gamma}_{N}\left(\hat{\eta}_{N}\right)
$$

where the cotangent vector field $\hat{\gamma}_{N} \in T^{*}\left(\hat{M}_{N}\right)$ reads as

$$
\hat{\gamma}_{N}\left(\hat{\eta}_{N}\right)=\left(\left.\frac{1}{N !} \frac{\partial^{N} \gamma^{T}\left(\hat{u}_{N}\right)}{\partial \varepsilon^{N}}\right|_{\varepsilon=0},\left.\frac{1}{(N-1) !} \frac{\partial^{N-1} \gamma^{T}\left(\hat{u}_{N}\right)}{\partial \varepsilon^{N-1}}\right|_{\varepsilon=0}, \cdots,\left.\frac{1}{1 !} \frac{\partial \gamma^{T}\left(\hat{u}_{N}\right)}{\partial \varepsilon}\right|_{\varepsilon=0}, \gamma^{T}\left(\eta_{0}\right)\right)^{T} .
$$

We hope that the cotangent vector field $\hat{\gamma}_{N}$ is a gradient field. If so, the potential function should be the following

$$
\begin{aligned}
& \hat{H}_{N}\left(\hat{\eta}_{N}\right)=\int_{0}^{1}<\hat{\gamma}_{N}\left(\lambda \hat{\eta}_{N}\right), \hat{\eta}_{N}>d \lambda \\
= & \int_{0}^{1} \sum_{i=0}^{N} \frac{1}{i !}<\left.\frac{\partial^{i} \gamma\left(\lambda \hat{u}_{N}\right)}{\partial \varepsilon^{i}}\right|_{\varepsilon=0}, \eta_{N-i}>d \lambda \\
= & \left.\frac{1}{N !} \frac{\partial^{N}}{\partial \varepsilon^{N}}\right|_{\varepsilon=0} \int_{0}^{1}<\gamma\left(\lambda \hat{u}_{N}\right), \hat{u}_{N}>d \lambda=\left.\frac{1}{N !} \frac{\partial^{N} H\left(\hat{u}_{N}\right)}{\partial \varepsilon^{N}}\right|_{\varepsilon=0} .
\end{aligned}
$$

Actually, the cotangent vector field $\hat{\gamma}_{N}$ is a gradient field. We can show that

$$
\hat{\gamma}_{N}\left(\hat{\eta}_{N}\right)=\frac{\delta \hat{H}_{N}\left(\hat{\eta}_{N}\right)}{\delta \hat{\eta}_{N}}
$$

According to the definition of the variational derivative, we have for any $S_{i} \in T\left(M\left(\eta_{i}\right)\right)$

$$
\begin{gathered}
\quad<\frac{\delta}{\delta \eta_{i}}\left(\left.\frac{1}{N !} \frac{\partial^{N} H\left(\hat{u}_{N}\right)}{\partial \varepsilon^{N}}\right|_{\varepsilon=0}\right), S_{i}\left(\eta_{i}\right)>=\left(\left.\frac{1}{N !} \frac{\partial^{N} H\left(\hat{u}_{N}\right)}{\partial \varepsilon^{N}}\right|_{\varepsilon=0}\right)^{\prime}\left(\eta_{i}\right)\left[S_{i}\left(\eta_{i}\right)\right] \\
=\left.\frac{1}{N !} \frac{\partial^{N}}{\partial \varepsilon^{N}}\right|_{\varepsilon=0} H^{\prime}\left(\hat{u}_{N}\right)\left[\varepsilon^{i} S_{i}\left(\eta_{i}\right)\right]=\left.\frac{1}{(N-i) !} \frac{\partial^{N-i}}{\partial \varepsilon^{N-i}}\right|_{\varepsilon=0} H^{\prime}\left(\hat{u}_{N}\right)\left[S_{i}\left(\eta_{i}\right)\right]
\end{gathered}
$$




$$
\begin{aligned}
& =\left.\frac{1}{(N-i) !} \frac{\partial^{N-i}}{\partial \varepsilon^{N-i}}\right|_{\varepsilon=0}<\frac{\delta H\left(\hat{u}_{N}\right)}{\delta \hat{u}_{N}}, S_{i}\left(\eta_{i}\right)>=\left.\frac{1}{(N-i) !} \frac{\partial^{N-i}}{\partial \varepsilon^{N-i}}\right|_{\varepsilon=0}<\gamma\left(\hat{u}_{N}\right), S_{i}\left(\eta_{i}\right)> \\
& =<\left.\frac{1}{(N-i) !} \frac{\partial^{N-i} \gamma\left(\hat{u}_{N}\right)}{\partial \varepsilon^{N-i}}\right|_{\varepsilon=0}, S_{i}\left(\eta_{i}\right)>, 0 \leq i \leq N .
\end{aligned}
$$

This shows that (3.19) holds, indeed. Therefore (3.17) is a Hamiltonian equation.

Let us now prove the property (3.16). Let $\beta=\frac{\delta H_{1}}{\delta u}, \gamma=\frac{\delta H_{2}}{\delta u} \in T^{*}(M)$. In virtue of (3.19), we can compute that

$$
\begin{aligned}
& \left\{\hat{H}_{1 N}, \hat{H}_{2 N}\right\}_{\hat{J}_{N}}\left(\hat{\eta}_{N}\right)=<\frac{\delta \hat{H}_{1 N}\left(\hat{\eta}_{N}\right)}{\delta \hat{\eta}_{N}}, \hat{J}_{N}\left(\hat{\eta}_{N}\right) \frac{\delta \hat{H}_{2 N}\left(\hat{\eta}_{N}\right)}{\delta \hat{\eta}_{N}}> \\
= & \sum_{i=0}^{N}<\left.\frac{1}{(N-i) !} \frac{\partial^{N-i} \beta\left(\hat{u}_{N}\right)}{\partial \varepsilon^{N-i}}\right|_{\varepsilon=0}, \sum_{j=N-i}^{N} \frac{1}{(i+j-N) !} \times \\
= & \left.\left.\frac{\partial^{i+j-N} J\left(\hat{u}_{N}\right)}{\partial \varepsilon^{i+j-N}}\right|_{\varepsilon=0} \frac{1}{(N-j) !} \frac{\partial^{N-j} \gamma\left(\hat{u}_{N}\right)}{\partial \varepsilon^{N-j}}\right|_{\varepsilon=0}> \\
= & \left.\frac{1}{N !} \frac{\partial^{N}}{\partial \varepsilon^{N}}\right|_{\varepsilon=0}<\beta\left(\hat{u}_{N}\right), J\left(\hat{u}_{N}\right) \gamma\left(\hat{u}_{N}\right)>=\left.\frac{1}{N !} \frac{\partial^{N-i}}{\partial \varepsilon^{N}}\right|_{\varepsilon=0}\left\{\hat{u}_{N}\right) \\
\partial \varepsilon^{N-i} & \left.\frac{1}{i !} \frac{\partial^{i}\left(J\left(\hat{u}_{N}\right) \gamma\left(\hat{u}_{N}\right)\right)}{\partial \varepsilon^{i}}\right\}_{J}\left(\hat{u}_{N}\right) .
\end{aligned}
$$

It follows that the equality (3.16) is true.

Further noting the concrete form of new Hamiltonian operators, a multi-Hamiltonian structure may readily be established for the perturbation equation. The proof is completed. $\#$

We should note that two important formulas: 3.15 and $(3.18) \cdot(3.15)$ provides a explicit formula for computing constants of motion of the $N$-th perturbation equations and (3.18) gives rise to an expression of perturbation cotangent vector fields.

\section{Applications to integrable equations}

\subsection{MKdV hierarchy}

We first consider the following MKdV hierarchy 25

$$
u_{t_{n}}=K_{n}=a_{n x}=J \Psi^{n} a_{0}=J \frac{\delta H_{n}}{\delta u}, n \geq 0,
$$

with

$$
J=\partial, \Psi(u)=-\frac{1}{4} \partial^{2}+u \partial^{-1} u \partial, H_{n}(u)=\frac{b_{n+1}-c_{n+1}}{2(2 n+1)}, n \geq 0,
$$


where $a_{i}, b_{i} c_{i}, i \geq 0$, are recursively defined by

$$
\left\{\begin{array}{l}
a_{0}=u, b_{0}=1, c_{0}=-1 \\
a_{i+1}=\Psi a_{i}, i \geq 0 \\
b_{i+1}=\frac{1}{2} a_{i x}+\partial^{-1} u \partial a_{i}, i \geq 0 \\
c_{i+1}=\frac{1}{2} a_{i x}-\partial^{-1} u \partial a_{i}, i \geq 0
\end{array}\right.
$$

and $\partial \partial^{-1}=\partial^{-1} \partial=1$. The first equation is exactly MKdV equation

$$
u_{t_{1}}=-\frac{1}{4} u_{x x x}+\frac{3}{2} u^{2} u_{x} .
$$

Its inverse scattering transform was first studied by Wadati26. The MKdV hierarchy 4.1) possesses zero curvature representations 25

$$
U_{t_{n}}-V_{x}^{(n)}+\left[U, V^{(n)}\right]=0, n \geq 0,
$$

with

$$
U=\left[\begin{array}{cc}
u & -\lambda \\
-1 & -u
\end{array}\right], V^{(n)}=\left[\begin{array}{cc}
\left(a \lambda^{n}\right)_{+} & \left(b \lambda^{n}\right)_{+} \lambda \\
\left(c \lambda^{n}\right)_{+} & -\left(a \lambda^{n}\right)_{+}
\end{array}\right], n \geq 0 .
$$

Here the plus symbol + stands for the choice of non-negative power of $\lambda$ and

$$
a=\sum_{i=0}^{\infty} a_{i} \lambda^{-i}, b=\sum_{i=0}^{\infty} b_{i} \lambda^{-i}, c=\sum_{i=0}^{\infty} c_{i} \lambda^{-i} .
$$

Applying the integrable theory in Sec. 3, we can obtain infinitely many new hereditary operators $\operatorname{per}_{N_{1}}$ per $_{N_{2}} \cdots \operatorname{per}_{N_{m}} \Phi, N_{1}, N_{2} \cdots, N_{m} \geq 1$, starting from the hereditary operator of MKdV hierarchy

$$
\Phi=\Psi^{\dagger}=-\frac{1}{4} \partial^{2}+\partial u \partial^{-1} u .
$$

We easily find that some explicit expressions:

$$
\begin{aligned}
& \left(\operatorname{per}_{N} \Phi\right)\left(\hat{\eta}_{N}\right)=\operatorname{diag}(\underbrace{-\frac{1}{4} \partial^{2}, \cdots,-\frac{1}{4} \partial^{2}}_{N+1})+\left[\sum_{k=0}^{i-j} \partial \eta_{k} \partial^{-1} \eta_{i-j-k}\right]_{(N+1) \times(N+1)}
\end{aligned}
$$

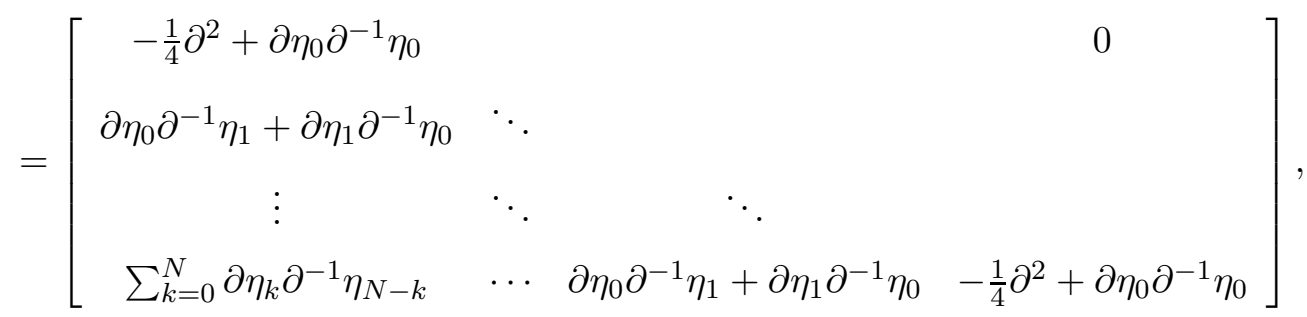

$$
\begin{aligned}
& \left(\operatorname{per}_{1} \operatorname{per}_{1} \Phi\right)(\eta)= \\
& {\left[\begin{array}{cccc}
-\frac{1}{4} \partial^{2}+\partial \eta_{0} \partial^{-1} \eta_{0} & 0 & 0 & 0 \\
\partial \eta_{0} \partial^{-1} \eta_{1}+\partial \eta_{1} \partial^{-1} \eta_{0} & -\frac{1}{4} \partial^{2}+\partial \eta_{0} \partial^{-1} \eta_{0} & 0 & 0 \\
\partial \eta_{0} \partial^{-1} \eta_{2}+\partial \eta_{2} \partial^{-1} \eta_{0} & 0 & -\frac{1}{4} \partial^{2}+\partial \eta_{0} \partial^{-1} \eta_{0} & 0 \\
\sum_{k=0}^{3} \partial \eta_{k} \partial^{-1} \eta_{3-k} & \partial \eta_{0} \partial^{-1} \eta_{2}+\partial \eta_{2} \partial^{-1} \eta_{0} & \partial \eta_{0} \partial^{-1} \eta_{1}+\partial \eta_{1} \partial^{-1} \eta_{0} & -\frac{1}{4} \partial^{2}+\partial \eta_{0} \partial^{-1} \eta_{0}
\end{array}\right]}
\end{aligned}
$$


where $\eta=\left(\eta_{0}, \eta_{1}, \eta_{2}, \eta_{3}\right)^{T}$. It needs a large amount of calculation if we directly prove the hereditariness of the above two operators.

The $N$-th order perturbation equation of $u_{t_{n}}=K_{n}$ reads as

$$
\hat{\eta}_{N t_{n}}=\left(\operatorname{per}_{N} K_{n}\right)\left(\hat{\eta}_{N}\right)=\left(\operatorname{per}_{N} \Phi\right)\left(\hat{\eta}_{N}\right)\left(\operatorname{per}_{N} K_{n-1}\right)\left(\hat{\eta}_{N}\right)=\left(\left(\operatorname{per}_{N} \Phi\right)\left(\hat{\eta}_{N}\right)\right)^{n} \hat{\eta}_{N x},
$$

among which is the $N$-th order perturbation equation of MKdV equation (4.2)

$$
\begin{aligned}
\eta_{i t_{1}} & =-\frac{1}{4} \partial^{2} \eta_{i x}+\sum_{l=0}^{i} \sum_{k=0}^{l} \partial \eta_{k} \partial^{-1} \eta_{l-k} \eta_{i-l, x} \\
& =-\frac{1}{4} \eta_{i x x x}+\frac{3}{2} \sum_{\substack{j+k+l=i \\
0 \leq j, k, l \leq i}} \eta_{j x} \eta_{k} \eta_{l}, 0 \leq i \leq N .
\end{aligned}
$$

The resulting local evolution equations defined by (4.5) are all integrable soliton ones for any $n, N \geq 1$. They all have zero curvature representations

$$
\left(\left(\operatorname{per}_{N} U\right)\left(\hat{\eta}_{N}\right)\right)_{t_{n}}-\left(\left(\operatorname{per}_{N} V^{(n)}\right)\left(\hat{\eta}_{N}\right)\right)_{x}+\left[\left(\operatorname{per}_{N} U\right)\left(\hat{\eta}_{N}\right),\left(\operatorname{per}_{N} V^{(n)}\right)\left(\hat{\eta}_{N}\right)\right]=0,
$$

and bi-Hamiltonian formulations

$$
\eta_{N t_{n}}=\left(\operatorname{per}_{N} J\right)\left(\hat{\eta}_{N}\right) \frac{\delta\left(\operatorname{per}_{N} H_{n}\right)\left(\hat{\eta}_{N}\right)}{\delta \hat{\eta}_{N}}=\left(\operatorname{per}_{N} M\right)\left(\hat{\eta}_{N}\right) \frac{\delta\left(\operatorname{per}_{N} H_{n-1}\right)\left(\hat{\eta}_{N}\right)}{\delta \hat{\eta}_{N}}
$$

where the Hamiltonian operator $M=J L=-\frac{1}{4} \partial^{3}+\partial u \partial^{-1} u \partial$. Therefore they possess infinitely many common symmetries $\operatorname{per}_{N} K_{m}, m \geq 0$, and infinitely many common constants of motion $\operatorname{per}_{N} H_{m}, m \geq 0$. In particular, for the $N$-th perturbation equation (4.6) of $\mathrm{MKdV}$ equation (4.2) we can get the following explicit results: two Hamiltonian functions

$$
\begin{aligned}
\left(\operatorname{per}_{N} H_{0}\right)\left(\hat{\eta}_{N}\right) & =\frac{1}{2} \sum_{j=0}^{N} \eta_{j} \eta_{N-j}, \\
\left(\operatorname{per}_{N} H_{1}\right)\left(\hat{\eta}_{N}\right) & =-\frac{1}{12} \sum_{j=0}^{N} \eta_{j} \eta_{N-j, x x}+\frac{1}{24} \sum_{j=0}^{N} \eta_{j x} \eta_{N-j, x} \\
& +\frac{1}{8} \sum_{\substack{j_{1}+j_{2}+j_{3}+j_{4}=N \\
0 \leq j_{1}, j_{2}, j_{3}, j_{4} \leq N}} \eta_{j_{1}} \eta_{j_{2}} \eta_{j_{3}} \eta_{j_{4}} ;
\end{aligned}
$$

a Hamiltonian pair

$$
\begin{gathered}
\left(\operatorname{per}_{N} J\right)\left(\hat{\eta}_{N}\right)=\operatorname{diag}(\underbrace{\partial, \partial, \cdots, \partial}_{N+1}) \\
\left(\operatorname{per}_{N} M\right)\left(\hat{\eta}_{N}\right)=\left[\begin{array}{cccc}
0 & & \\
& & -\frac{1}{4} \partial^{3}+\partial \eta_{0} \partial^{-1} \eta_{0} \partial \\
& \vdots & \sum_{k=0}^{1} \partial \eta_{k} \partial^{-1} \eta_{1-k} \partial \\
-\frac{1}{4} \partial^{3}+\partial \eta_{0} \partial^{-1} \eta_{0} \partial & \sum_{k=0}^{1} \partial \eta_{k} \partial^{-1} \eta_{1-k} \partial & \cdots & \sum_{k=0}^{N} \partial \eta_{k} \partial^{-1} \eta_{N-k} \partial
\end{array}\right] ;
\end{gathered}
$$


and a pair of Lax operators

$$
\left(\operatorname{per}_{N} U\right)\left(\hat{\eta}_{N}\right)=\left[\begin{array}{cccc}
A_{0} & & & 0 \\
A_{1} & A_{0} & & \\
\vdots & \ddots & \ddots & \\
A_{N} & \cdots & A_{1} & A_{0}
\end{array}\right],\left(\operatorname{per}_{N} V^{(1)}\right)\left(\hat{\eta}_{N}\right)=\left[\begin{array}{cccc}
B_{0} & & & 0 \\
B_{1} & B_{0} & & \\
\vdots & \ddots & \ddots & \\
B_{N} & \cdots & B_{1} & B_{0}
\end{array}\right],
$$

where the operators $A_{i}, B_{i}, 0 \leq i \leq N$, are given by

$$
\begin{aligned}
& A_{i}=\left[\begin{array}{cc}
\eta_{i} & \lambda \\
-1 & -\eta_{i}
\end{array}\right], \quad B_{i}= \\
& {\left[\begin{array}{ccc}
\eta_{i} \lambda-\frac{1}{4} \eta_{i x x}+\frac{1}{2} \sum_{\substack{j_{1}+j_{2}+j_{3}=i \\
0 \leq j_{1}, j_{2}, j_{3} \leq i}} \eta_{j_{1}} \eta_{j_{2}} \eta_{j_{3}} & \delta_{i 0} \lambda^{2}+\frac{1}{2}\left(\eta_{i x}+\sum_{j=0}^{i} \eta_{j} \eta_{i-j}\right) \lambda \\
-\delta_{i 0} \lambda+\frac{1}{2}\left(\eta_{i x}-\sum_{j=0}^{i} \eta_{j} \eta_{i-j}\right) & -\eta_{i} \lambda+\frac{1}{4} \eta_{i x x}-\frac{1}{2} \sum_{\substack{j_{1}+j_{2}+j_{3}=i \\
0 \leq j_{1}, j_{2}, j_{3} \leq i}} \eta_{j_{1}} \eta_{j_{2}} \eta_{j_{3}}
\end{array}\right] .}
\end{aligned}
$$

In addition, we can generate a $\tau$-symmetry algebra 27] of the perturbation equation (4.5) by a perturbation of $\tau$-symmetries of $u_{t_{n}}=K_{n}$. Moreover we may also consider the nonlinear problem of the Lax systems

$$
\hat{\phi}_{N x}=\left(\operatorname{per}_{N} U\right)\left(\hat{\eta}_{N}\right) \hat{\phi}_{N}, \hat{\phi}_{N t_{n}}=\left(\operatorname{per}_{N} V^{(n)}\right)\left(\hat{\eta}_{N}\right) \hat{\phi}_{N},
$$

similar to Ref. [25].

\subsection{Kadomtsev-Petviashvili equation}

Let us now consider the Kadomtsev-Petviashvili equation

$$
u_{t}=K(u)=K_{3}(u)=\partial_{x}^{-1} u_{y y}-u_{x x x}-6 u u_{x} .
$$

It has Lax pair

$$
L(u)=\frac{i}{\sqrt{3}} \partial_{y}+\partial_{x}^{2}+u, A(u)=3 i \partial_{x}^{-1} u_{y}-3 u_{x}-6 u \partial_{x}-4 \partial_{x}^{3}, i=\sqrt{-1}
$$

and time independent symmetries

$$
K_{1}=\frac{3}{2} u_{x}, K_{2}=u_{y}, K_{n}=\left[K_{n-1}, \tau\right], n \geq 2,
$$

where $\tau=y K_{3}+\frac{2}{3} x u_{y}+\frac{4}{3} \partial_{x}^{-1} u_{y}$ and further the $k$-th order master symmetry (see for example Ref. [28])

$$
\tau^{(k)}=y^{k-1}, \tau_{i_{1} i_{2} \cdots i_{j}}^{(k)}=\operatorname{ad}_{K_{i_{1}}} \operatorname{ad}_{K_{i_{2}}} \cdots \operatorname{ad}_{K_{i_{j}}} y^{k+j-1}, j, k \geq 1 .
$$

Therefore KP equation (4.7) possesses the following time polynomial dependent symmetries

$$
\sigma^{(k)}=\sum_{l=0}^{k} \frac{t^{l}}{l !}\left(\operatorname{ad}_{K}\right)^{l} \tau^{(k)}, \sigma_{i_{1} i_{2} \cdots i_{j}}^{(k)}=\sum_{l=0}^{k} \frac{t^{l}}{l !}\left(\operatorname{ad}_{K}\right)^{l} \tau_{i_{1} i_{2} \cdots i_{j}}^{(k)}, j, k \geq 1,
$$


which contain the symmetries with the forms $\frac{\partial}{\partial t} \sigma^{(k)}$ and $\frac{\partial}{\partial t} \sigma_{i_{1} i_{2} \cdots i_{j}}^{(k)}$.

The corresponding perturbation equation $\hat{\eta}_{N t}=\hat{K}_{N}$ becomes

$$
\eta_{i t}=\partial_{x}^{-1} \eta_{i y y}-\eta_{i x x x}-6 \sum_{j=0}^{i} \eta_{j} \eta_{i-j, x}, 0 \leq i \leq N .
$$

Following the general theory in Sec. 3, (4.9) is also an integrable equation in $2+1$ dimensions. For example, it has Lax pair $\left(\hat{L}_{n}\left(\hat{\eta}_{N}\right)\right)_{t}=\left[\hat{A}_{N}\left(\hat{\eta}_{N}\right), \hat{L}_{N}\left(\hat{\eta}_{N}\right)\right]$, where the spectral operator $\hat{L}_{N}$ and the Lax operator $\hat{A}_{N}$ read as

$$
\begin{aligned}
& \hat{L}_{N}=\left[\begin{array}{cccc}
\frac{i}{\sqrt{3}} \partial_{y}+\partial_{x}^{2}+\eta_{0} & & 0 & \\
\eta_{1} & \frac{i}{\sqrt{3}} \partial_{y}+\partial_{x}^{2}+\eta_{0} & & \\
\vdots & \ddots & \ddots & \\
\eta_{N} & \cdots & \eta_{1} & \frac{i}{\sqrt{3}} \partial_{y}+\partial_{x}^{2}+\eta_{0}
\end{array}\right] \\
& \hat{A}_{N}=\left[\begin{array}{cccc}
A\left(\eta_{0}\right) & & 0 & \\
3 i \partial_{x}^{-1} \eta_{1 y}-3 \eta_{1 x}-6 \eta_{1} \partial_{x} & A\left(\eta_{0}\right) & & \\
\vdots & \ddots & \ddots & A\left(\eta_{0}\right)
\end{array}\right] .
\end{aligned}
$$

Moreover (4.9) has the $k$-th order master symmetry

$$
\operatorname{per}_{N} \tau^{(k)}=(y^{k-1}, \underbrace{0, \cdots, 0}_{N})^{T}, k \geq 1,
$$

and thus possesses time polynomial dependent symmetries

$$
\operatorname{per}_{N} \sigma^{(k)}=\sum_{l=0}^{k} \frac{t^{l}}{l !}\left(\operatorname{adper}_{N} K\right)^{l} \operatorname{per}_{N} \tau^{(k)}, k \geq 1,
$$

and

$$
\operatorname{per}_{N} \sigma_{i_{1} i_{2} \cdots i_{j}}^{(k)}=\sum_{l=0}^{k} \frac{t^{l}}{l !}\left(\operatorname{adper}_{N} K\right)^{l} \operatorname{per}_{N} \tau_{i_{1} i_{2} \cdots i_{j}}^{(k)}, j, k \geq 1
$$

Although KP equation has no regular recursion operator 29, we may also construct a biHamiltonian formulation of (4.9) through the perturbation of the bi-Hamiltonian one 30 . But here we omitted the discussion because of the complicated notation.

Let us now take another form of the Kadomtsev-Petviashvili equation

$$
u_{x t}=\partial_{x} K=u_{y y}-\left(u_{x x x}+6 u u_{x}\right)_{x}
$$

Evidently its solutions include all solutions of the Kadomtsev-Petviashvili equation (4.7) and hence it is a little more general than (4.7). We are about to see that there exists a 
different symmetry property between (4.7) and (4.10). Now the corresponding linearized equation to (4.10) is as follows

$$
\sigma_{x t}=\partial_{x}\left(K^{\prime}[\sigma]\right)=\partial_{y y} \sigma-\partial_{x}^{4} \sigma-6 \partial_{x}^{2}(u \sigma) .
$$

One may directly prove that 4.10) possesses the following three time dependent symmetries

$$
\begin{aligned}
\sigma^{(1)}(f)= & -\frac{1}{6} f_{t}+f u_{x}, \\
\sigma^{(2)}(f)= & -\frac{1}{12} f_{t t} y+\frac{1}{2} f_{t} y u_{x}+f u_{y}, \\
\sigma^{(3)}(f)= & -\frac{1}{36} f_{t t t} y^{2}+f_{t t}\left(\frac{1}{6} y^{2} u_{x}-\frac{1}{18} x\right) \\
& +f_{t}\left(\frac{2}{3} u+\frac{2}{3} y u_{y}+\frac{1}{3} x u_{x}\right)+f u_{t},
\end{aligned}
$$

with an arbitrary function $f$ of $t$, each of which is not any symmetry of (4.7) while $f_{t t} \neq$ $0, f_{t t t} \neq 0$ or $f_{t t t t} \neq 0$ respectively. That kind of symmetries is first introduced in Ref. [31]. In fact, there is a rule to generate these symmetries. For example,

$$
\sigma^{(2)}(f)=-\frac{1}{12} f_{t t} y+f_{t}\left[K,-\frac{1}{12} y\right]+f\left[K,\left[K,-\frac{1}{12} y\right]\right] .
$$

We can also construct new vector fields

$$
\sigma^{(n)}(f)=\sum_{i=0}^{n} \frac{d^{n-i} f}{d t^{n-i}}\left(\operatorname{ad}_{K}\right)^{i} y^{n-1}, n \geq 4 .
$$

But in these vector fields, there exist nonlinear terms involving $u_{y}$ or $u$ etc., which can't be balanced in the linearized equation (4.11). Therefore (4.15) are not any symmetries of (4.10). The symmetries determined by (4.12), (4.13) and (4.14) constitute a Lie algebra

$$
\left\{\begin{aligned}
{\left[\sigma^{(1)}(f), \sigma^{(1)}(g)\right] } & =0 \\
{\left[\sigma^{(2)}(f), \sigma^{(2)}(g)\right] } & =\sigma^{(1)}\left(\frac{1}{2} f g_{t}-\frac{1}{2} f_{t} g\right), \\
{\left[\sigma^{(3)}(f), \sigma^{(3)}(g)\right] } & =\sigma^{(3)}\left(f g_{t}-f_{t} g\right) \\
{\left[\sigma^{(1)}(f), \sigma^{(2)}(g)\right] } & =0 \\
{\left[\sigma^{(1)}(f), \sigma^{(3)}(g)\right] } & =\sigma^{(1)}\left(\frac{1}{3} f g_{t}-f_{t} g\right), \\
{\left[\sigma^{(2)}(f), \sigma^{(3)}(g)\right] } & =\sigma^{(2)}\left(\frac{2}{3} f g_{t}-f_{t} g\right),
\end{aligned}\right.
$$

where $f, g$ are two functions of $t$. Note that the second commutator relation is somewhat different from one given in Ref. [32]. But if we choose $\tilde{\sigma}^{(2)}(f)=\sqrt{2} \sigma^{(2)}(f)$, then two algebras are the same. The above symmetry algebra contains the following three subalgebras $\left\{\sigma^{(1)}(f), \sigma^{(2)}(f)\right\},\left\{\sigma^{(3)}(f)\right\}$ and $\left\{\sigma^{(1)}(f), \sigma^{(2)}(f), \sigma^{(3)}(1)=u_{t}\right\}$. The last subalgebra has the simple commutator relations

$$
\left[u_{t}, \sigma^{(1)}(f)\right]=\sigma^{(1)}\left(f_{t}\right),\left[u_{t}, \sigma^{(2)}(f)\right]=\sigma^{(2)}\left(f_{t}\right) .
$$


It is easy to see that the $N$-th order perturbation equation of 4.10

$$
\eta_{i x t}=\eta_{i y y}-\left(\eta_{i x x x}+6 \sum_{j=0}^{i} \eta_{j} \eta_{i-j, x}\right)_{x}, 0 \leq i \leq N
$$

has also the similar symmetry property. More precisely, it possesses the following time dependent symmetries with an arbitrary function of $t$

$$
\begin{aligned}
\operatorname{per}_{N} \sigma^{(1)}(f)= & \left(-\frac{1}{6} f_{t}+f \eta_{0 x}, f \eta_{1 x}, \cdots, f \eta_{N x}\right)^{T}, \\
\operatorname{per}_{N} \sigma^{(2)}(f)= & \left(-\frac{1}{12} f_{t t} y+\frac{1}{2} f_{t} y \eta_{0 x}+f \eta_{0 y}, \frac{1}{2} f_{t} y \eta_{1 x}+f \eta_{1 y}, \cdots, \frac{1}{2} f_{t} y \eta_{N x}+f \eta_{N y}\right)^{T}, \\
\operatorname{per}_{N} \sigma^{(3)}(f)= & \left(-\frac{1}{36} f_{t t t} y^{2}+f_{t t}\left(\frac{1}{6} y^{2} \eta_{0 x}-\frac{1}{18} x\right)+f_{t}\left(\frac{2}{3} \eta_{0}+\frac{2}{3} y \eta_{0 y}+\frac{1}{3} x \eta_{0 x}\right)+f \eta_{0 t},\right. \\
& f_{t t}\left(\frac{1}{6} y^{2} \eta_{1 x}-\frac{1}{18} x\right)+f_{t}\left(\frac{2}{3} \eta_{1}+\frac{2}{3} y \eta_{1 y}+\frac{1}{3} x \eta_{1 x}\right)+f \eta_{1 t}, \\
& \left.\cdots, f_{t t}\left(\frac{1}{6} y^{2} \eta_{N x}-\frac{1}{18} x\right)+f_{t}\left(\frac{2}{3} \eta_{N}+\frac{2}{3} y \eta_{N y}+\frac{1}{3} x \eta_{N x}\right)+f \eta_{N t}\right)^{T},
\end{aligned}
$$

which constitute the same infinite dimensional symmetry algebra as (4.16). In fact, the equation (4.17) has the same integrable property as (4.10). It is an interesting problem how to construct integrable equations which possess a kind of symmetries involving an arbitrary function of time variable.

It is well known that the evolution equation has not a similar property, i.e. it doesn't possess 33 the following symmetries involving an arbitrary function $f$ of the time variable

$$
\sigma^{(n)}=\sum_{i=0}^{n} \frac{d^{n-i} f}{d t^{n-i}} S_{i}(u)
$$

where $S_{i}, 0 \leq i \leq n$, don't depend explicitly on the time variable. In general, it possesses time polynomial dependent symmetries generated by its master symmetries 34 . Therefore (4.7) and (4.10) have different symmetry algebras.

\section{Concluding remarks}

We may also make another perturbation series

$$
\check{u}_{N}=\sum_{i=0}^{N} \frac{\varepsilon^{i}}{i !} \xi_{i}
$$

similar to the perturbation series (1.12). This moment, the corresponding perturbation equation reads as

$$
\check{\xi}_{N t}=\check{K}_{N}\left(\check{\xi}_{N}\right), \check{\xi}_{N}=\left(\xi_{0}^{T}, \xi_{1}^{T}, \cdots, \xi_{N}^{T}\right)^{T},
$$

where the perturbation vector field is of the form

$$
\check{K}_{N}\left(\check{\xi}_{N}\right)=\left(K^{T}\left(\xi_{0}\right),\left.\frac{\partial K^{T}\left(\check{u}_{N}\right)}{\partial \varepsilon}\right|_{\varepsilon=0}, \cdots,\left.\frac{\partial^{N} K^{T}\left(\check{u}_{N}\right)}{\partial \varepsilon^{N}}\right|_{\varepsilon=0}\right)^{T} .
$$


In an analogous way, we can generate another new hereditary operator

$$
\check{\Phi}_{N}\left(\check{\xi}_{N}\right)=\left[\left.\left(\begin{array}{c}
i \\
j
\end{array}\right) \frac{\partial^{i-j} \Phi\left(\check{u}_{N}\right)}{\partial \varepsilon^{i-j}}\right|_{\varepsilon=0}\right]_{i, j=0,1, \cdots, N}
$$

from a known hereditary operator $\Phi(u)$, and $\check{\Phi}_{N}\left(\check{\xi}_{N}\right)$ is a recursion operator of (5.2) provided that $\Phi(u)$ is a recursion operator of the original equation $u_{t}=K(u)$. From the above expression, we see that the formation of the perturbation equation under the perturbation series (5.1) is simpler but the corresponding recursion operator is more complicated than ones under (1.12). However there exists an intimate relation between two kinds of perturbations because we have a Miura transformation

$$
\eta_{i}=\eta_{i}\left(\xi_{0}, \xi_{1}, \cdots, \xi_{N}\right)=\frac{1}{i !} \xi_{i}, 0 \leq i \leq N .
$$

For example, the operator $\breve{\Phi}_{N}\left(\check{\xi}_{N}\right)$ may be generated by a transformation

$$
\begin{aligned}
& \check{\Phi}_{N}\left(\check{\xi}_{N}\right)=\frac{\partial \check{\xi}_{N}}{\partial \hat{\eta}_{N}} \hat{\Phi}_{N}\left(\hat{\eta}_{N}\right)\left(\frac{\partial \check{\xi}_{N}}{\partial \hat{\eta}_{N}}\right)^{-1} \\
& =\left[\begin{array}{ccccc}
1 & & & & 0 \\
& 1 ! & & & \\
& 2 ! & & \\
& & & \ddots & \\
0 & & & & N !
\end{array}\right] \hat{\Phi}_{N}\left(\hat{\eta}_{N}\right)\left[\begin{array}{ccccc}
1 & & & & 0 \\
& 1 ! & & & \\
& & 2 ! & & \\
& & \ddots & \\
0 & & & N !
\end{array}\right]^{-1} \text {. }
\end{aligned}
$$

In fact, we can similarly obtain any new tensors by means of the Miura transformation (5.4). For instance, a new Hamiltonian operator may be engendered by

$$
\check{J}_{N}\left(\check{\xi}_{N}\right)=\frac{\partial \check{\xi}_{N}}{\partial \hat{\eta}_{N}} \hat{J}_{N}\left(\hat{\eta}_{N}\right)\left(\frac{\partial \check{\xi}_{N}}{\partial \hat{\eta}_{N}}\right)^{\dagger}=\left[\left.\frac{i ! j !}{(i+j-N) !} \frac{\partial^{i+j-N} J\left(\check{u}_{N}\right)}{\partial \varepsilon^{i+j-N}}\right|_{\varepsilon=0}\right]_{i, j=0,1, \cdots, N} .
$$

We remark that by the resulting perturbation equations in Sec. 3, we can generate approximate solutions of the original equations to a precision $o\left(\varepsilon^{N}\right)$. This is different from the construction of the $\tau$ functions in bilinear formation, where the expansion series holds exactly for any order precision. It is also of interest to note that the perturbation equations are all integrable coupling with the original equations and the original ones always appear in the first position. Therefore our integrable theory provides an approach for constructing integrable coupling of soliton equations and enriches the intention of perturbation bundle established in Ref. 18]. However it is still a problem deserving of investigation how to construct more general integrable coupling by perturbation.

Acknowledgments: One of the authors (W. X. Ma) would like to thank the Alexander von Humboldt Foundation, the National Natural Science Foundation of China and the Shanghai Science and Technology Commission of China for their financial support. He has benefited from helpful discussions with Dr. W. Oevel. 


\section{References}

[1] V. I. Karpman, Soliton evolution in the presence of perturbation, Phys. Scr. 20, 462-478 (1979).

[2] J. P. Keener and D. W. McLaughlin, Solitons under perturbations, Phys. Rev. A 16, 777-790 (1977).

[3] Y. Kodama and M. J. Ablowitz, Perturbations of solitons and solitary waves, Stud. Appl. Math. 64, 225-245 (1981).

[4] P. J. Olver, Hamiltonian perturbation theory and water waves, Contemporary Mathematics 28, 231-249 (1984).

[5] R. L. Herman, A direct approach to studying soliton perturbations, J. Phys. A: Math. Gen. 23, 2327-2362 (1990).

[6] M. Tanaka, Perturbations on the K-dV equation - An approach based on the multiple time scale expansion, J. Phys. Soc. Jpn. 49, 807-812 (1980).

[7] R. Grimshaw and H. Mitsudera, Slowly varying solitary wave solutions of the perturbed Korteweg-de Vries equation revisited, Stud. Appl. Math. 90, 75-86 (1993).

[8] J. N. Elgin, T. Brabec and S. M. J. Kelly, A perturbative theory of soliton propagation in the presence of the third order dispersion, Optics Commun. 114, 321-328 (1995).

[9] G. J. Ballantyne, P. T. Gough and D. P. Taylor, Deriving average soliton equations with a perturbation method, Phys. Rev. E 5, 825-828 (1995).

[10] C. Currò, A. Donato and A. Ya. Povzner, Perturbation method for a generalized Burger's equation, Intern. J. Nonlinear Mechanics 27, 149-155 (1992).

[11] Y. Matsuno, Multisoliton perturbation theory for the Benjamin-Ono equation and its application to real physical systems, Phys. Rev. E 51, 1471-1483 (1995).

[12] Y. S. Kivshar and B. A. Malomed, Dynamics of solitons in nearly integrable systems, Rev. Mod. Phys. 61, 763-915 (1989).

[13] K. M. Tamizhmani and M. Lakshmanan, Complete integrability of the Kortewegde Vries equation under perturbation around its solution: Lie-Bäcklund symmetry approach, J. Phys. A: Math. Gen. 16, 3773-3782 (1983).

[14] W. X. Ma and B. Fuchssteiner, The bi-Hamiltonian structure of the perturbation equations of KdV hierarchy, Phys. Lett. A 213, 49 (1996).

[15] R. A. Kraenkel, M. A. Manna and J. G. Pereira, The Korteweg-de Vries hierarchy and long water-waves, J. Math. Phys. 36, 307-320 (1995).

[16] R. A. Kraenkel, M. A. Manna, J. C. Montero and J. G. Pereira, Boussinesq solitarywave as a multiple-time solution of the Korteweg-de Vries hierarchy, preprint, 1995.

[17] B. Fuchssteiner and A. S. Fokas, Symplectic structures, their Bäcklund transformations and hereditary symmetries, Physica D 4 47-66 (1981). 
[18] B. Fuchssteiner, Coupling of completely integrable systems: the perturbation bundle, Applications of Analytic and Geometric Methods to Nonlinear Differential Equations, edited by P. A. Clarkson, Kluwer, Dordrecht, 125-138 (1993).

[19] W. Oevel, Dirac constraints in the field theory: lifts of Hamiltonian systems to the cotangent bundle, J. Math. Phys. 29, 210-219 (1988).

[20] W. X. Ma, Poisson manifolds and classical Hamiltonian operators, Northeastern Mathematical Journal 6, 346-356 (1990).

[21] B. Fuchssteiner, Application of hereditary symmetries to nonlinear evolution equations, Nonlinear Analysis TMA 3 849-862 (1979).

[22] I. M. Gel'fand and I. Y. Dorfman, Hamiltonian operators and algebraic structures related to them, Funct. Anal. Appl. 13 248-262 (1979).

[23] F. Magri, A simple model of the integrable Hamiltonian equation, J. Math. Phys. 19, 1156-1162 (1978).

[24] H. H. Chen and J. E. Lin, On the integrability of multidimensional nonlinear evolution equations, J. Math. Phys. 28, 347-350 (1987).

[25] W. X. Ma, Symmetry constraint of MKdV equations by binary nonlinearization, Physica A 219, 467-481 (1995).

[26] M. Wadati, The modified Korteweg-de Vries equation, J. Phys. Soc. Jpn. 34, 12891296 (1973).

[27] W. X. Ma, $K$-symmetries and $\tau$-symmetries of evolution equations and their Lie algebras, J. Phys. A: Math. Gen. 23, 2707-2716 (1990).

[28] W. X. Ma, The algebraic structures of isospectral Lax operators and applications to integrable equations, J. Phys. A: Math. Gen. 25, 5329-5343 (1992).

[29] V. E. Zakharov and B. G. Konopelchenko, On the theory of recursion operator, Commun. Math. Phys. 94, 483-509 (1984).

[30] I. Ya. Dorfman and A. S. Fokas, Hamiltonian theory over noncommutative rings and integrability in multidimensions, J. Math. Phys. 33, 2504-2514 (1992).

[31] F. Schwarz, Symmetries of the two-dimensional Korteweg-de Vries equation, J. Phys. Soc. Jpn. 51, 2387-2388 (1982).

[32] D. David, N. Kamran, D. Levi and P. Winternitz, Symmetry reduction for the Kadomtsev-Petviashvili equation using a loop algebra, J. Math. Phys. 27, 1225-1237 (1986).

[33] W. X. Ma, The generators of vector fields and the time dependent symmetries of evolution equations, Science in China A 34, 769-782 (1991).

[34] B. Fuchssteiner, Master symmetries, higher order time-dependent symmetries and conserved densities of nonlinear evolution equations, Prog. Theor. Phys. 70, 15081522 (1983). 